\title{
Dynamic Resilient Containment Control in Multi-Robot Systems
}

\author{
Matteo Santilli, Mauro Franceschelli, Member, IEEE, Andrea Gasparri, Senior Member, IEEE
}

\begin{abstract}
In this paper we study the dynamic resilient containment control problem for continuous-time multi-robot systems (MRSs), i.e., the problem of designing a local interaction protocol that drives a set of robots, namely the followers, toward a region delimited by the positions of another set of robots, namely the leaders, under the presence of adversarial robots in the network. In our setting, all robots are anonymous, i.e., they do not recognize the identity or class of other robots. We consider as adversarial all those robots that intentionally or accidentally try to disrupt the objective of the multi-robot system, e.g., robots that are being hijacked by a cyber-physical attack or have experienced a fault. Under specific topological conditions defined by the notion of $(r, s)$-robustness, our control strategy is proven to be successful in driving the followers toward the target region, namely a hypercube, in finite-time. It is also proven that the followers cannot escape the moving containment area despite the persistent influence of anonymous adversarial robots. Numerical results with a team of 44 robots are provided to corroborate the theoretical findings.
\end{abstract}

Index Terms-Containment control, distributed algorithms, large scale networks, multi-robot systems, resilient protocols.

\section{INTRODUCTION}

$\mathbf{M}$ ULTI-Agent Systems (MASs) consist in large scale networks of autonomous dynamical systems, capable of observing the environment through their sensors and reacting to it using their actuators. The ultimate goal of a MAS is to cooperate in order to reach a global objective that the single agent is not capable of reaching individually. Thanks to their capability to model large networks of mobile robots or swarms, in the last decades they gained a lot of interest both in the academic and industrial environments, see for instance [1]-[3]. Multi-agent systems that involve robotic agents and/or swarms are commonly known as Multi-Robot Systems (MRSs). One of the great strengths of MRSs is the relatively small cost of deployment of a large number of heterogeneous robotics units that can fulfill tasks that the humans would not be able to do or that would be too dangerous for them, e.g.,

Matteo Santilli and Andrea Gasparri are with the Department of Engineering, Roma Tre University, 00146 Rome, Italy, (e-mail: matteo.santilli@uniroma3.it, gasparri@dia.uniroma3.it).

Mauro Franceschelli is corresponding author and is with the Department of Electrical and Electronic Engineering, University of Cagliari, 09123 Cagliari, Italy (e-mail: mauro.franceschelli@unica.it).

This work was partially supported by "Convenzione triennale tra la Fondazione di Sardegna e gli Atenei Sardi - Regione Sardegna L.R. 7/2007 annualità 2019-DGR DGR 28/21 del 17.05.2015" with project "Formal Methods and Technologies for the Future of Energy Systems", CUP F72F20000350007, call 2019.

This work was partially supported by the project "PARADISE - Precision fARming for sustAinable proDuctIon in Suburban arEas", Avviso Pubblico "Progetti Strategici" per l'Area di Specializzazione "Green Economy", POR FESR 2014-2020, CUP F84D20000030003. environmental exploration or search and rescue operations in hostile environments like caves or areas subjected to severe weather. Indeed, MRSs are envisioned to be exploited in futuristic scenarios where a large number of robots provides resiliency, flexibility and cost reduction. One of such futuristic applications consist of satellite swarms which find strength in numbers by being able to continue their operation and provide service coverage despite outages, faults, or attacks [4]. Another futuristic application consists in swarms of autonomous drones that thanks to eventual improvements of their intelligence enable a single pilot to control hundreds of them [5].

In order to design an effective and reliable MRS it is necessary to address various challenges. One of the most important ones definitely regards the locality of information, i.e., the robotic agents in the network usually possess or can communicate data only with agents which are close to them, known as neighbors, hence great effort has to be devoted into the design of distributed protocols, which enable the MRS to achieve the desired objective using only local sensing and communication. Another great challenge consists in being able to design a MRS able to keep working under faults of sensor or actuators, or even under cyber-physical attacks. While it is generally true that there is strength in numbers, it is also true, unfortunately, that most formal models of MRSs show that the behavior that emerges from the local interactions of agents through sensing or communication is extremely vulnerable to the malfunction of single agents, i.e., a single agent can disrupt, accidentally or intentionally, the emergent behavior of a large scale network by executing movements different from the nominal control action. In this work we aim to design a distributed control protocol able to achieve the global objective under the presence of adversarial agents, which may arise from either faults or intentional cyber-physical attacks on the robotic network.

\section{A. Literature Review}

In this work we address the dynamic resilient containment control problem, i.e., the problem of letting a set of followers reach and then remain confined within an area defined by the positions of a set of possibly moving leaders, under the existence of adversarial agents, i.e., robots that may have suffered a cyber-attack or may have lost the capability of executing the prescribed control protocol.

In the last decade, the containment problem has received a lot of interest in the research community with several studies such as [6]-[10] that address both the static and the dynamic versions of the problem. The static containment 
control problem considers a containment region that is fixed in space since the leaders that define it are stationary whereas the dynamic version involves leaders that can possibly evolve and move in their domain. The static version has been investigated in [6] where the authors propose a distributed control strategy for a team of unicycles able to drive the followers inside the convex hull of a set of static leaders while also preserving the connectivity of the interactions among the agents in the network. Another example is [7] where the authors developed an average consensus protocol that is also capable of achieving the static containment problem under directed topology. A hybrid containment problem has been studied in [8] where the authors in the context of partial difference equations and Laplacian feedback proposed a stop-and-go policy to move the followers while keeping intermittently the containment region active. In [9] the authors consider instead a Lyapunov based approach applied to linear local interaction protocols under fixed and switching network topologies that is able to drive the followers to a convex hull defined by the positions of the leaders in both the static and dynamic version of the problem. The two different contexts of the containment problem are addressed also in [10] where the authors provide a sliding mode controller for a second order multi-agent system that is proven to asymptotically reach a containment area when the leaders are static whereas in the case of dynamic leaders with bounded input the followers are only guaranteed to stay close to the containment area.

In recent years, great efforts have also been devoted to the study of secure distributed protocols [11] $-[15]$. In [11] the authors propose a distributed algorithm to asymptotically estimate a measurement under the presence of limited sensor attacks in the network. A different approach is proposed in [12], [13] where the authors introduce a new notion of topology robustness and a new distributed protocol, known as W-MSR algorithm, that is able to solve the consensus protocol under such topological conditions. The basic idea of the W-MSR protocol consists in collecting the local information of the neighbors and then discarding the data coming from the farthest neighbors. This kind of approach and notion of robustness became quite popular at the state of the art, with recent examples as [16]-[20]. For example, the author in [16] developed a slightly edited version of the W-MSR protocol to achieve resilient consensus for a hybrid multi-agent system composed of both continuous time and discrete time dynamical agents whereas in [17] the authors analyze the triangular and square lattice robot formation interactions in order to guarantee the necessary robustness to achieve resilient consensus.

In this work, our proposed robust interaction protocol builds upon the notion of robustness introduced in [12], [13]. Applications of secure protocols to the containment problem are still relatively scarce with recent example like [21], [22] that address the output resilient containment problem. In [21] the authors propose a distributed observer-based protocol that is able to guarantee the uniformly ultimately boundedness of the tracking error under the presence of adversarial agents and sensor faults. In [22] instead the authors consider the case of signal attacks on the dynamics of the followers and propose an approach based on reinforcement learning to solve the problem after it has been converted in an equivalent $H_{\infty}$ tracking problem. An alternative research direction to network robustness is to develop protocols based on fault detection and identification. These methods rely on the possibility to detect the adversarial agents, exploiting the presence of external agents [23] or requiring strict topological conditions [24].

Our distributed protocol is a discontinuous control action based on the sign function. At the state of the art, many works [25]-[32] have proposed distributed protocols based on sign function able to solve consensus and optimization problems. Just to mention a few, in [25] the authors proposed four similar finite-time protocols for a high order multi-agent system able to reach consensus in the case of a leaderless network or able to track the leaders if present. In [26] distributed optimization problems for convex functions have instead been addressed. The authors were able to prove that their algorithm is either able to reach consensus in finite-time while asymptotically minimizing the cost functional or either they are able to prove the finite-time convergence for both. Distributed control strategies that use sign function have also been shown to have a certain degree of robustness with respect to disturbances [33]-[35] and uncooperative agents [36]-[38]. In [33] the authors introduced a distributed protocol for a second order multi-agent system able to reach consensus in finite-time under the presence of bounded disturbances while also maintaining the initial topology. A finite-time consensus on the median value is analyzed in [36], [39] where the authors showed that the proposed protocol is also robust to uncooperative agents if the network possesses a topological property known as $k$-safety.

Finally, our convergence analysis is based on non-smooth Lyapunov analysis. We refer the reader to $[40]-[45]$ for a comprehensive overview on the topic.

\section{B. Main Contribution}

In this paper, we consider a distributed sign-based local interaction protocol for a first order multi-robot system that has already been studied in the literature for the consensus problem, e.g. [28]-[30], and characterize its properties in regard to the dynamic resilient containment problem. The MRS is proven to be robust against adversarial robotic agents if certain topological conditions, known as $(r, s)$-robustness, are met. In particular, we characterize a sufficient graph theoretical condition that is able to guarantee the achievement of the dynamic resilient containment of the followers in the network.

\section{Assumptions on the multi-robot system:}

1) The robots in the network are all anonymous, i.e., incapable of discerning if their neighbors are followers, leaders, or adversarial agents.

2) The robots do not have access to absolute positions (GPS).

3) The robots are able measure the presence of their neighbors with respect to their common bearing directions, i.e., each agent can pinpoint the exact orthant in which each neighbor lies with respect to the common bearing directions and with respect to its own reference frame. 
4) The robots share $d-1$ common bearing directions. Methods to achieve common bearings exist, such as the one presented in [46], but require measurement of relative distance and bearing angles instead of just presence.

5) The dynamics of the position of each robot can be approximated by a continuous-time $d$-dimensional integrator. Each follower and leader have bounded velocity.

In our preliminary work presented in [47] we addressed the static containment problem under the presence of adversarial agents in the network, providing a conservative graph theoretical condition, denoted as $(k, \mathcal{S})$-connectivity, that enables a control law based on the sign function to achieve the desired objective. In this work, as opposed to [47], we propose and characterize several properties of a different and much less conservative graph theoretical condition based on the notion of $(r, s)$-robustness, while also considering the case of dynamic leaders, i.e., robot leaders can move with bounded speed. Furthermore, we extend our results to consider the case of a time-varying switching graph.

\section{Structure of the paper}

The paper is organized as follows, in Section $\Pi$ fundamental results on non-smooth analysis and preliminaries on the modeling of the network are given. In Section III the dynamic resilient containment control problem is formally stated and the local interaction protocol is presented. In Section IV the convergence properties of the proposed control strategy are discussed. In Section $\mathrm{V}$ numerical simulations that illustrate the effectiveness of the results are presented and finally in Section VI concluding remarks are given.

\section{Preliminaries}

\section{A. Non-smooth analysis}

In this section, we review some fundamental results concerning non-smooth analysis that will be used hereafter in the paper to investigate the convergence properties of the considered distributed protocol.

Consider the (possibly discontinuous) dynamical system

$$
\dot{x}=f(x), \quad x \in \mathbb{R}^{n}, \quad x(0)=x_{0} \in \mathbb{R}^{n},
$$

where $f(x): \mathbb{R}^{n} \rightarrow \mathbb{R}^{n}$, is defined almost everywhere, i.e., it is defined for every $x \in \mathbb{R}^{n} \backslash W$, where $W$ is a subset of $\mathbb{R}^{n}$ of measure zero. Furthermore, $f(x)$ is measurable in an open region $Q \subset \mathbb{R}^{n}$ and for all compact sets $D \subset Q$ there exists a constant $A_{D}$ such that $\|f(x)\| \leq A_{D}$ almost everywhere in $D$.

If the differential equation (1) has discontinuous right-hand side, following [45] we understand the corresponding solution in the so-called Filippov sense as the solution of an appropriate differential inclusion, as explained in the next definition.

Definition 1 (Filippov Solution). A vector function $x(\cdot)$ is called solution of (1) on a time interval $\left[t_{0}, t_{i}\right]$ if $x(\cdot)$ is absolutely continuous on $\left[t_{0}, t_{i}\right]$ and for almost all $t \in\left[t_{0}, t_{i}\right]$

$$
\dot{x} \in K[f](x),
$$

where $K[f](x): \mathbb{R}^{n} \rightarrow 2^{\mathbb{R}^{n}}$ is defined as

$$
K[f](x) \triangleq \bigcap_{\delta>0} \bigcap_{\mu\{H\}=0} \overline{\operatorname{co}}\{f(B(x, \delta) \backslash H)\},
$$

where $\bigcap_{\mu\{H\}=0}$ denotes the intersection over all sets $H$ of Lebesgue measure zero, $B(x, \delta)$ the ball of radius $\delta$ centered at $x, \overline{\text { co }}$ the convex closure, and $2^{n}$ the set of subsets of $\mathbb{R}^{n}$.

Briefly, the idea of the Filippov's solution is that the tangent vector to a solution, where it exists, must lie in the convex closure of the values of the vector field in progressively smaller neighborhoods around the solution point. A very important aspect of this definition is given by the possibility of discarding sets of measure zero. Indeed, this technical detail allows solutions to be defined even at points where the vector field itself is not defined. If $f(x)$ is measurable and locally bounded then the set-valued map $K[f](x)$ is upper semicontinuous, compact, convex valued and locally bounded so that the differential inclusion (2) possesses a Filippov solution for each initial condition $x_{0}$.

Let us now recall the definition of the Clarke's Generalized Gradient.

Definition 2 (Clarke's Generalized Gradient [44]). Let $V(x): \mathbb{R}^{n} \rightarrow \mathbb{R}$ be a locally Lipschitz continuous function. Its Clarke's generalized gradient $\partial V(x)$ is defined as

$$
\partial V(x) \triangleq c o\left\{\lim _{i \rightarrow \infty} \nabla V\left(x_{i}\right) \mid x_{i} \rightarrow x, x_{i} \notin \Omega_{V} \cup N\right\},
$$

where $\nabla V$ denotes the conventional gradient, $x_{i} \in \mathbb{R}^{n}$ represents a point of an infinite succession which converges to $x \in \mathbb{R}^{n}$ as $i$ grows to infinity, $\Omega_{V}$ is a set of Lebesgue measure zero which contains all points where $\nabla V(x)$ does not exist, and $N$ is an arbitrary set of measure zero.

We now review the chain rule which allows to differentiate Lipschitz regular functions along the Filippov's solution trajectories.

Theorem 1 (Chain Rule [42]). Let $x(\cdot)$ be a Filippov solution to (1) and $V: \mathbb{R}^{n} \rightarrow \mathbb{R}$ be a Lipschitz and, in addition, regular function. Then $V(x)$ is absolutely continuous, $(d / d t) V(x(t))$ exists almost everywhere and

$$
\frac{d}{d t} V(x(t)) \in^{\text {a.e. }} \dot{\tilde{V}}(x),
$$

where the set-valued Lie derivative $\dot{\tilde{V}}(x)$ is defined as

$$
\dot{\tilde{V}}(x) \triangleq \bigcap_{\xi \in \partial V(x(t))} \xi^{T} K[f](x) .
$$

Let us now recall a revised version of the Generalized Lyapunov theorem given in [41] based on the results given in [42]. This will prove useful to establish finite-time stability results for dynamical systems described by differential equations with discontinuous right-hand side.

Theorem 2 (Finite-Time Stability Theorem). Let $x(\cdot)$ be $a$ Filippov solution to (1) and $V(x): \mathbb{R}^{n} \rightarrow \mathbb{R}$, be a time independent regular function such that $V(x)=0 \forall x \in \mathcal{C}(t)$ and $V(x)>0 \quad \forall x \notin \mathcal{C}(t)$, with $\mathcal{C}(t) \in \mathbb{R}^{n}$ a compact set. Let $x: \mathbb{R} \rightarrow \mathbb{R}^{n}$ and $V(x(t))$ be absolutely continuous on $\left[t_{0}, \infty\right)$ 


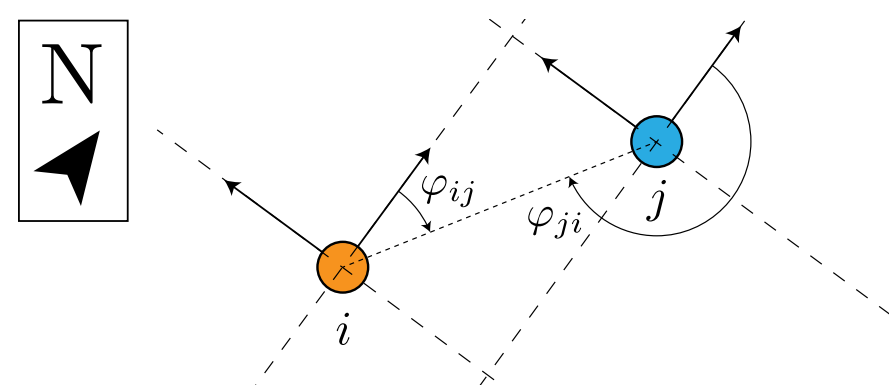

Figure 1. Example of sensing of presence between robots $i$ and $j$ utilizing bearing angle measurements among robots.

with $d / d t(V(x)) \leq-\mu<0$ with $\mu>0$ for $\forall x \notin \mathcal{C}(t)$. Then, $V(x(t))$ converges to 0 in finite-time and $x(t)$ reaches the compact set $\mathcal{C}(t)$ in finite-time as well.

At this point, let us now define the discontinuous "sign" function, the discontinuous and set-valued "SIGN" function and the discontinuous vector "sign" function as follows

$$
\begin{aligned}
& \operatorname{sign}(y)=\left\{\begin{aligned}
1, & \text { if } \quad y>0, \\
0, & \text { if } \quad y=0, \\
-1, & \text { if } \quad y<1,
\end{aligned} \quad y \in \mathbb{R}\right. \\
& \operatorname{SIGN}(y) \in\left\{\begin{array}{cl}
1 & \text { if } y>0, \\
{[-1,1]} & \text { if } y=0, \quad y \in \mathbb{R}, \\
-1 & \text { if } y<0 .
\end{array}\right. \\
& \operatorname{sign}(y)=\left[\operatorname{sign}\left(y_{1}\right), \cdots, \operatorname{sign}\left(y_{n}\right)\right]^{T}, \quad y \in \mathbb{R}^{n}
\end{aligned}
$$

\section{B. Robot and Network Modeling}

Let $\mathcal{G}=(\mathcal{V}, \mathcal{E})$ be an undirected graph, where $\mathcal{V}=\{1, \ldots, n\}$ is the set of robotic agents and $\mathcal{E} \subseteq\{\mathcal{V} \times \mathcal{V}\}$ is the set of edges representing their ability to sense each other. More precisely, an edge $(i, j)$ belongs to the set $\mathcal{E}$ if robot $i$ can sense the presence of robot $j$. In addition, let $\mathcal{N}_{i}=\{j \in \mathcal{V}:(i, j) \in \mathcal{E}\}$ be the set of neighbors of robot $i$. Note that in a $d$ dimensional Euclidean space, with $d>0$, sensing of presence under the assumption of $d-1$ common bearing directions can be realized for example with bearing angle measurements with respect to the $d-1$ common bearing directions. Figure 1 depicts a scenario in $d=2$ where a common north bearing direction is shared between robot $i$ and robot $j$. The bearing angle measurements with respect to the common north, represented here by $\varphi_{i j}$ and $\varphi_{j i}$, allow the robots to pinpoint the quadrants (orthants in generic multidimensional spaces) in which their neighbors lie with respect to their own reference frames. We want also to point out that since the interactions among the robots are encoded by a sensing graph, the robots are not susceptible to spoofing attacks and similar threats that exploit exchanges of messages.

Let $x_{i}(t)=\left[x_{i, 1}(t), \ldots, x_{i, d}(t)\right]^{T} \in \mathbb{R}^{d}$ be the state of the robot $i$ representing its position where $x_{i, p}(t)$ denotes the $p$-th component of the state $x_{i}(t)$. In addition, let us assume that the robots evolve with the single integrator dynamics, i.e., $\dot{x}_{i}(t)=u_{i}(t), i \in \mathcal{V}$ where $u_{i}(t) \in \mathbb{R}^{d}$ is the local control input to be later specified. Let us also recall the definition of the infinity norm $\|\cdot\|_{\infty}$ that is defined as the largest among the components absolute value of a vector, i.e., $\left\|x_{i}(t)\right\|_{\infty}=\max _{p \in\{1, \ldots, d\}}\left\{\left|x_{i, p}(t)\right|\right\}$.

A path in a graph $\mathcal{G}$ is a sequence of consecutive edges connecting two nodes. A graph is said to be connected if there exists a path between any pair of nodes. A cut is a partition of the node set $\mathcal{V}$ in two disjoint subsets $\mathcal{S}_{1}, \mathcal{S}_{2} \subset \mathcal{V}$ which are joined by at least one edge.

We now generalize the notion of cut for couples of disjoint subsets of the node set $\mathcal{V}$ with the next definition of generalized cut set.

Definition 3 (Generalized cut set). Consider an undirected graph $\mathcal{G}=(\mathcal{V}, \mathcal{E})$ and two non-empty disjoint node subsets $\mathcal{S}_{1}, \mathcal{S}_{2} \subset \mathcal{V}$. Let the generalized cut set of $\mathcal{S}_{1}$ and $\mathcal{S}_{2}$, denoted as $\left(\mathcal{S}_{1}, \mathcal{S}_{2}\right)$, be defined as the set of edges with one endpoint in $\mathcal{S}_{1}$ and the other in $\mathcal{S}_{2}$, i.e., $\left(\mathcal{S}_{1}, \mathcal{S}_{2}\right)=\left\{(i, j) \in \mathcal{E}: i \in \mathcal{S}_{1}, j \in \mathcal{S}_{2}\right\}$.

In the rest of the paper we denote the number of edges belonging to the generalized cut set $\left(\mathcal{S}_{1}, \mathcal{S}_{2}\right)$ as $\left|\left(\mathcal{S}_{1}, \mathcal{S}_{2}\right)\right|$.

We now report the notions of reachability and robustness introduced in [12] for the case of directed graphs and adapted here for the case of undirected graphs.

Definition 4 ( $r$-reachable set). Consider an undirected graph $\mathcal{G}=(\mathcal{V}, \mathcal{E})$ with node set $\mathcal{V}$, edge set $\mathcal{E}$, and a non-empty node subset $\mathcal{S} \subset \mathcal{V}$. We say that $\mathcal{S}$ is an r-reachable set if $\exists i \in \mathcal{S}$ such that $\left|\mathcal{N}_{i} \backslash \mathcal{S}\right| \geq r$, where $r \in \mathbb{Z}_{\geq 0}$.

Basically, a subset of nodes $\mathcal{S} \subset \mathcal{V}$ is $r$-reachable according to Definition 4 if there exists at least one node in set $\mathcal{S}$ with at least $r$ neighbors outside set $\mathcal{S}$. Extending the concept of $r$-reachable sets to a graph we obtain the next definition.

Definition 5 ( $r$-robust graph). Consider an undirected graph $\mathcal{G}=(\mathcal{V}, \mathcal{E})$ with node set $\mathcal{V}$ and edge set $\mathcal{E}$. We say that $\mathcal{G}$ is r-robust if for all pairs of non-empty, disjoint subsets $\mathcal{S}_{1}, \mathcal{S}_{2} \subset \mathcal{V}$, at least one of $\mathcal{S}_{1}$ or $\mathcal{S}_{2}$ is $r$-reachable, where $r \in \mathbb{Z}_{\geq 0}$.

Notably, Definition 5 implies that at anytime in an $r$-robust graph there is always a set that contains a node that possesses at least $r$ neighbors outside its set. This definition, however, does not give any insight on how many nodes are satisfying the reachability property. To improve the redundancy of information in robust graphs, the same authors in [13] introduced the notion of $(r, s)$-reachability.

Definition $6((r, s)$-reachable set). Consider an undirected graph $\mathcal{G}=(\mathcal{V}, \mathcal{E})$ with node set $\mathcal{V}$, edge set $\mathcal{E}$, and a nonempty node subset $\mathcal{S} \subset \mathcal{V}$. We say that $\mathcal{S}$ is an $(r, s)$-reachable set if there are at least $s$ nodes in $\mathcal{S}$ that possesses $r$ or more neighbors outside the set $\mathcal{S}$, where $r, s \in \mathbb{Z}_{\geq 0}$.

Defining the set $\mathcal{X}_{\mathcal{S}}^{r}$ as the set of nodes within the subset $\mathcal{S}$ that have at least $r$ neighbors outside $\mathcal{S}$, i.e., $\mathcal{X}_{\mathcal{S}}^{r}=\left\{i \in \mathcal{S}:\left|\mathcal{N}_{i} \backslash \mathcal{S}\right| \geq r\right\}$, then Definition 6 is satisfied when $\left|\mathcal{X}_{\mathcal{S}}^{r}\right| \geq s$. Notice that $r$-reachability is equivalent to $(r, 1)$-reachability so the notion of $(r, s)$-reachability is a generalization of the concept of $r$-reachability. Now, ex- 


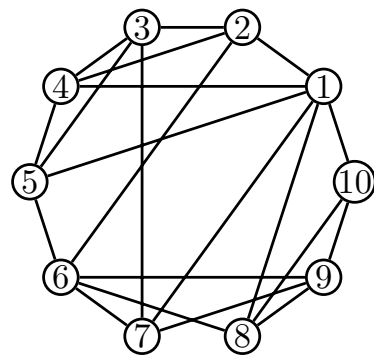

Figure 2. Example of a graph with 10 nodes that is $(2,2)$-robust.

tending this definition to a graph we obtain the notion of $(r, s)$-robustness.

Definition $7((r, s)$-robust graph). Consider an undirected graph $\mathcal{G}=(\mathcal{V}, \mathcal{E})$ with node set $\mathcal{V}$ and edge set $\mathcal{E}$. We say that $\mathcal{G}$ is $(r, s)$-robust with $r \in \mathbb{Z}_{\geq 0}$ and $1 \leq s \leq|\mathcal{V}|$ if for all pairs of non-empty, disjoint subsets $\mathcal{S}_{1}, \mathcal{S}_{2} \subset \mathcal{V}$, at least one of following holds:

1) $\left|\mathcal{X}_{\mathcal{S}_{1}}^{r}\right|=\left|\mathcal{S}_{1}\right|$, i.e., all nodes in $\mathcal{S}_{1}$ are $r$-reachable;

2) $\mathcal{X}_{\mathcal{S}_{2}}^{r}=\left|\mathcal{S}_{2}\right|$, i.e., all nodes in $\mathcal{S}_{2}$ are $r$-reachable;

3) $\left|\mathcal{X}_{\mathcal{S}_{1}}^{r}\right|+\left|\mathcal{X}_{\mathcal{S}_{2}}^{r}\right| \geq s$.

As for the concept of reachability, $(r, 1)$-robustness is equivalent to $r$-robustness. Generally speaking, a graph that is $(r, s)$-robust is also $\left(r, s^{\prime}\right)$-robust with $1 \leq s^{\prime} \leq s$. Furthermore, any $(r, s)$-robust graph is clearly connected. The reader is referred to [13] for more insight and properties of $(r, s)$-robust graphs.

In Figure 2 it is depicted a $(2,2)$-robust graph composed of 10 nodes. It can be verified by checking every pairs of nonempty disjoint subsets of $\{1, \ldots, 10\}$ that one of the three conditions introduced in Definition 7 is always satisfied. It can also be seen that this graph is not $(3,1)$-robust since for example the sets $\mathcal{S}_{1}=\{2,3,4\}$ and $\mathcal{S}_{2}=\{8,9,10\}$ do not have a single node with three or more neighbors outside their respective sets. Regarding the notion of $(r, s)$-reachability we can consider for example the set $\mathcal{S}_{3}=\{4,5,6\}$ and notice that this set is $(3,2)$-reachable since nodes 4 and 6 have both at least three neighbors outside $\mathcal{S}_{3}$ while node 5 has only two.

\section{Problem Setting}

Let us consider a multi-robot system composed of three different sets of robotic agents, namely followers, leaders and adversarial agents. More specifically, the set of followers, denoted as $\mathcal{V}_{f}$, represents the agents that execute the considered control protocol. Furthermore, the set of leaders, denoted as $\mathcal{V}_{\ell}$, represents the agents which define the containment area that the followers are expected to reach and not escape from. Finally, the set of adversarial agents, denoted as $\mathcal{V}_{a}$, represents the agents that either purposely or unintentionally may disrupt the desired behaviour of the system. Clearly the node set $\mathcal{V}$ of the network $\mathcal{G}$ is such that $\mathcal{V}=\mathcal{V}_{f} \cup \mathcal{V}_{\ell} \cup \mathcal{V}_{a}$, with $\mathcal{V}_{f}, \mathcal{V}_{\ell}$, and $\mathcal{V}_{a}$ disjoint sets. Moreover, we assume that the sets $\mathcal{V}_{f}$ and $\mathcal{V}_{\ell}$ are non-empty. In the rest of the paper, the following additional set $\mathcal{V}_{f \ell}=\mathcal{V}_{f} \cup \mathcal{V}_{\ell}$ is used to denote the set of followers and leaders together.
In particular, the state of the followers, leaders, and adversarial agents have respectively the following dynamics

$$
\begin{aligned}
\dot{x}_{i}(t) & =u_{i}^{f}(t), & & i \in \mathcal{V}_{f}, \\
\dot{x}_{i}(t) & =u_{i}^{\ell}(t), \quad\left\|u_{i}^{\ell}(t)\right\|_{\infty} \leq u_{\max }^{\ell}, & & i \in \mathcal{V}_{\ell}, \\
\dot{x}_{i}(t) & =u_{i}^{a}(t), & & i \in \mathcal{V}_{a}
\end{aligned}
$$

where $u_{i}^{f}(t) \in \mathbb{R}^{d}$ is the local control input of the follower $i \in \mathcal{V}_{f}, u_{i}^{\ell}(t) \in \mathbb{R}^{d}$ is the local control input of the leader $i \in \mathcal{V}_{\ell}$, and $u_{i}^{a}(t) \in \mathbb{R}^{d}$ is the local control input of the adversarial agent $i \in \mathcal{V}_{a}$. We assume that the control inputs $u_{i}^{f}(t), u_{i}^{\ell}(t)$, and $u_{i}^{a}(t)$ are all locally essentially bounded. Moreover, we assume that the input of the leaders is upper bounded by a positive scalar $u_{\max }^{\ell} \in \mathbb{R}_{>0}$.

Remark 1 (Adversarial agents' behaviour). The state of the adversarial agents, as the other kinds of agents, evolve under the assumption of locally essentially boundedness of their control inputs. This requirement is related to the physical nature of our MRS in which the states of the robots represent their positions, hence requiring their state to be absolutely continuous. Furthermore, we consider the worst case scenario where the adversarial agents may influence the network with arbitrary behavior, thus including the case in which they have full knowledge of the network state and cooperate among them to disrupt the desired behaviour of the MRS.

In the sequel, for the sake of analysis and without loss of generality we collect the robots by sorting and labelling them according to their kind. Note that this labeling is only for the sake of our theoretical analysis; the agents are anonymous and thus are not able to discern the kind or identity of their neighboring agents. Thus, we denote the stacked vector of the followers' state with $\mathbf{x}^{f}=\left[x_{1}^{T}, \ldots, x_{n_{f}}^{T}\right]^{T}$ where $n_{f}=\left|\mathcal{V}_{f}\right|$, the stacked vector of the leaders' state with $\mathbf{x}^{\ell}=\left[x_{n_{f}+1}^{T}, \ldots, x_{n_{f}+n_{\ell}}^{T}\right]^{T}$ where $n_{\ell}=\left|\mathcal{V}_{\ell}\right|$, and the stacked vector of the adversarial agents' state with $\mathbf{x}^{a}=\left[x_{n_{f}+n_{\ell}+1}^{T}, \ldots, x_{n_{f}+n_{\ell}+n_{a}}^{T}\right]^{T}$ where $n_{a}=\left|\mathcal{V}_{a}\right|$ and $n_{f}+n_{\ell}+n_{a}=n$. In addition, for the sake of the analysis we will denote the stacked vector of followers and leaders state as $\mathbf{x}=\left[\mathbf{x}^{f^{T}} \mathbf{x}^{\ell^{T}}\right]^{T}$. Furthermore, we will collect the $p$-th component of the states of the followers and leaders in the vector $\mathbf{x}_{p}=\left[x_{1, p}, \ldots, x_{n_{f}+n_{\ell}, p}\right]^{T}$.

Our goal is to characterize a cooperative distributed protocol $u_{i}^{f}(t)$ to be executed by the followers $\mathcal{V}_{f}$ so that they reach in finite-time $T$ the containment region $\mathcal{C}\left(\mathbf{x}_{\ell}(t)\right)$ defined according to the position of the leaders $\mathcal{V}_{\ell}$ and remain inside in it for all $t \geq T$, despite the influence of the adversarial agents. In particular, the containment area that the followers will have to reach and then remain within is a hypercube defined as

$$
\mathcal{C}\left(\mathbf{x}^{\ell}(t)\right)=\left\{y \in \mathbb{R}^{d}: y_{p} \in\left[\underline{x}_{p}(t), \bar{x}_{p}(t)\right], p \in\{1, \ldots, d\}\right\}
$$

where the terms $\bar{x}_{p}(t)$ and $\underline{x}_{p}(t)$ represent the maximum and minimum value among the $p$-th component of the leaders as

$$
\begin{aligned}
& \bar{x}_{p}(t)=\max _{i \in \mathcal{V}_{\ell}} x_{i, p}(t), \\
& \underline{x}_{p}(t)=\min _{i \in \mathcal{V}_{\ell}} x_{i, p}(t) .
\end{aligned}
$$


We are now ready to formalize our problem.

Problem 1. Let us consider a containment region $\mathcal{C}\left(\mathbf{x}_{\ell}(t)\right)$ defined according to the location of the leaders as in eq. (13). Our goal is to characterize a distributed cooperative control law $u_{i}^{f}(t)$ for the followers so that for any initial condition $\mathbf{x}^{f}(0), \mathbf{x}^{\ell}(0), \mathbf{x}^{a}(0)$, and any locally essentially bounded input of the anonymous adversarial agents $u_{i}^{a}(t)$ with $i \in \mathcal{V}_{a}$, the following holds true

$$
x_{i}(t) \in \mathcal{C}\left(\mathbf{x}^{\ell}(t)\right), i \in \mathcal{V}_{f}, \forall t \geq T,
$$

with $T$ the finite convergence time.

Next, we provide the distributed cooperative control law $u_{i}^{f}(t)$, implemented by each follower $i \in \mathcal{V}_{f}$, that solves Problem 1 under proper graph theoretical conditions on the graph $\mathcal{G}$ :

$$
u_{i}^{f}(t)=-\alpha \sum_{j \in \mathcal{N}_{i}} \operatorname{sign}\left(x_{i}(t)-x_{j}(t)\right) \quad i \in \mathcal{V}_{f},
$$

with $\alpha \in \mathbb{R}_{>0}$ a positive gain.

We recall that the control law in eq. (17) has already been studied at the state of the art in works such as [28]-[30] for the consensus problem. We are instead going to consider this control law in the context of the dynamic resilient containment control problem under $(r, s)$-robust networks.

Since the local interaction law (17) is implemented on independent axes, the robots are only required to sense the presence of neighbors with respect to the common bearing directions on their own right-handed reference frame in order to execute it. Furthermore, the robots need to share only $d-1$ common bearing direction since the $d$-th direction can be evaluated by computing an orthogonal direction with respect to the other common bearing directions.

We also point out that except for sensing of presence, each robot does not perform any other operation such as ordering, filtering, or scaling before executing the control law (17). This is in contrast to many works on resilient control that rely on i) filtering out data coming from the farthest neighbors and ii) weighting the filtered data according to some nonnegative scalar. In our approach we do not scale the states of the neighbors because the sign function is unaffected by the magnitude of the states' differences. Moreover, we do not filter out portions of the data because under the topological conditions considered in Theorem 3 it is shown that the sign of the sum of the sign functions exploited in the local interactions, i.e., the resulting direction in which the followers move, is unaffected by the behaviour of the adversarial agents.

In the next section, we theoretically characterize:

i) the topological conditions that are able to guarantee the robustness of the proposed control protocol (17);

ii) the finite-time required to the followers to reach the containment area $\mathcal{C}\left(\mathrm{x}^{\ell}(t)\right)$ defined in eq. (13).

\section{TheORETICAL ANALYSis}

Before analyzing the theoretical properties of the proposed control strategy let us first introduce the following topological results on a $(r, s)$-robust graph $\mathcal{G}$.
Lemma 1. Consider an undirected $(r, s)$-robust graph $\mathcal{G}=(\mathcal{V}, \mathcal{E})$. Consider three disjoint subsets $\mathcal{V}_{1}, \mathcal{V}_{2}$, and $\mathcal{V}_{3}$ such that $\mathcal{V}=\mathcal{V}_{1} \cup \mathcal{V}_{2} \cup \mathcal{V}_{3}$, and $\mathcal{V}_{1}, \mathcal{V}_{2}$ are non-empty. Let $r>\left|\mathcal{V}_{3}\right|$. Then it holds

$$
\left|\left(\mathcal{V}_{1}, \mathcal{V}_{2}\right)\right| \geq\left(r-\left|\mathcal{V}_{3}\right|\right) \min \left\{\left|\mathcal{V}_{1}\right|,\left|\mathcal{V}_{2}\right|, s\right\},
$$

where $\left|\left(\mathcal{V}_{1}, \mathcal{V}_{2}\right)\right|$ is the cardinality of the generalized cut set $\left(\mathcal{V}_{1}, \mathcal{V}_{2}\right)$ in Definition 3

Proof. Since the graph is $(r, s)$-robust and $\mathcal{V}_{1}, \mathcal{V}_{2}$ are nonempty, disjoint subsets of $\mathcal{V}$, by denoting $\mathcal{X}_{\mathcal{V}_{1}}^{r} \subseteq \mathcal{V}_{1}$ and $\mathcal{X}_{\mathcal{V}_{2}}^{r} \subseteq \mathcal{V}_{2}$ the subsets of $r$-reachable nodes within $\mathcal{V}_{1}$ and $\mathcal{V}_{2}$, it holds (see Definition 7) that at least one of the following three conditions is verified:

1) $\left|\mathcal{X}_{\mathcal{V}_{1}}^{r}\right|=\left|\mathcal{V}_{1}\right|$, i.e., all nodes in $\mathcal{V}_{1}$ are r-reachable;

2) $\mathcal{X}_{\mathcal{V}_{2}}^{r}=\left|\mathcal{V}_{2}\right|$, i.e., all nodes in $\mathcal{V}_{2}$ are r-reachable;

3) $\left|\mathcal{X}_{\mathcal{V}_{1}}^{r}\right|+\left|\mathcal{X}_{\mathcal{V}_{2}}^{r}\right| \geq s$.

Now, we compute the cardinality of the generalized cut set $\left|\left(\mathcal{V}_{1}, \mathcal{V}_{2}\right)\right|$, i.e., the number of edges with one endpoint in $\mathcal{V}_{1}$ and the other in $\mathcal{V}_{2}$ (see Definition 3 ).

- If 1) holds, i.e., $\left|\mathcal{X}_{\mathcal{V}_{1}}^{r}\right|=\left|\mathcal{V}_{1}\right|$, then $\left|\mathcal{V}_{1}\right| \geq s$. Since $r>\left|\mathcal{V}_{3}\right|$ it follows that there are at least $\left(r-\left|\mathcal{V}_{3}\right|\right)\left|\mathcal{V}_{1}\right|$ edges connecting sets $\mathcal{V}_{1}$ and $\mathcal{V}_{2}$, thus $\left|\left(\mathcal{V}_{1}, \mathcal{V}_{2}\right)\right| \geq\left(r-\left|\mathcal{V}_{3}\right|\right)\left|\mathcal{V}_{1}\right|$

- If 2) holds, i.e., $\left|\mathcal{X}_{\mathcal{V}_{2}}^{r}\right|=\left|\mathcal{V}_{2}\right|$, then $\left|\mathcal{V}_{2}\right| \geq s$. Since $r>\left|\mathcal{V}_{3}\right|$ it follows that there are at least $\left(r-\left|\mathcal{V}_{3}\right|\right)\left|\mathcal{V}_{2}\right|$ edges connecting sets $\mathcal{V}_{1}$ and $\mathcal{V}_{2}$, thus $\left|\left(\mathcal{V}_{1}, \mathcal{V}_{2}\right)\right| \geq\left(r-\left|\mathcal{V}_{3}\right|\right)\left|\mathcal{V}_{2}\right|$

- If 3) holds, i.e., $\left|\mathcal{X}_{\mathcal{V}_{1}}^{r}\right|+\left|\mathcal{X}_{\mathcal{V}_{2}}^{r}\right| \geq s$, then $\left|\mathcal{V}_{1}\right|+\left|\mathcal{V}_{2}\right| \geq s$. Since $r>\left|\mathcal{V}_{3}\right|$ and there are at least $s \quad r$-reachable nodes in $\mathcal{V}_{1} \cup \mathcal{V}_{2}$ it follows that there are at least $\left(r-\left|\mathcal{V}_{3}\right|\right) s$ edges connecting sets $\mathcal{V}_{1}$ and $\mathcal{V}_{2}$, thus $\left|\left(\mathcal{V}_{1}, \mathcal{V}_{2}\right)\right| \geq\left(r-\left|\mathcal{V}_{3}\right|\right) s$

It easily follows that, in all cases, it holds

$$
\left|\left(\mathcal{V}_{1}, \mathcal{V}_{2}\right)\right| \geq\left(r-\left|\mathcal{V}_{3}\right|\right) \min \left\{\left|\mathcal{V}_{1}\right|,\left|\mathcal{V}_{2}\right|, s\right\},
$$

hence proving the statement of this lemma.

As it will be shown later, this result guarantees the existence of a certain number of connections between the two cooperative sets of robots in the network, i.e., the followers and the leaders, allowing us to prove the effectiveness of the local interaction protocol introduced in eq. (17) in regard to Problem 1

We are now ready to state our result concerning the finitetime convergence of the proposed distributed protocol introduced in eq. (17).

Theorem 3. Consider a multi-robot system consisting of $n_{f}$ followers, $n_{\ell}$ leaders, and $n_{a}$ adversarial agents and with network topology encoded by an undirected graph $\mathcal{G}=(\mathcal{V}, \mathcal{E})$. Let the dynamics of the robots be described by eqs. (10), (11), and (12) under the assumption of locally essentially boundedness of their control inputs. Moreover, let the control inputs of the leaders be bounded by $u_{\max }^{\ell} \in \mathbb{R}_{\geq 0}$, i.e., $\left\|u_{i}^{\ell}(t)\right\|_{\infty} \leq u_{\max }^{\ell}$. Let the followers execute the control protocol in eq. 17]. If graph $\mathcal{G}$ is $(r, s)$-robust, and if

$$
\begin{gathered}
\left(\alpha-u_{\max }^{\ell}\right)\left(r-n_{a}\right) \min \left\{n_{f}, n_{\ell}, s\right\}>\alpha\left|\left(\mathcal{V}_{f}, \mathcal{V}_{a}\right)\right|, \\
\alpha>u_{\max }^{\ell}, \quad \text { and } \quad r>n_{a},
\end{gathered}
$$


where $\alpha$ is the positive gain in eq. 177) and $\left|\left(\mathcal{V}_{f}, \mathcal{V}_{a}\right)\right|$ is the cardinality of a generalized cut-set as in Definition 3 then there exists a finite-time $T>0$ such that

$$
x_{i}(t) \in \mathcal{C}\left(\mathbf{x}^{\ell}(t)\right), \quad \forall i \in \mathcal{V}_{f}, \quad \forall t \geq T,
$$

where the containment region $\mathcal{C}\left(\mathrm{x}^{\ell}(t)\right)$ is defined as in eq. (13).

Proof. The main steps of the proof are hereinafter provided. The complete proof is reported in Appendix A.

First we consider the evolution of the system along its $p$-th component, then we generalize the result to hold in a complete $d$-dimensional space. To this end, we consider the following non-smooth Lyapunov candidate function

$$
V_{p}\left(\mathbf{x}_{p}(t)\right)=V_{p}^{M}\left(\mathbf{x}_{p}(t)\right)+V_{p}^{m}\left(\mathbf{x}_{p}(t)\right),
$$

where the terms $V_{p}^{M}\left(\mathbf{x}_{p}(t)\right)$ and $V_{p}^{m}\left(\mathbf{x}_{p}(t)\right)$ express the distance between the farthest robots and the leaders at the maximum and minimum values $\bar{x}_{p}(t)$ and $\underline{x}_{p}(t)$ as

$$
\begin{aligned}
V_{p}^{M}\left(\mathbf{x}_{p}(t)\right) & =\sum_{i \in \mathcal{I}_{p}^{M}\left(\mathbf{x}_{p}(t)\right)}\left|x_{i, p}(t)-\bar{x}_{p}(t)\right|, \\
V_{p}^{m}\left(\mathbf{x}_{p}(t)\right) & =\sum_{i \in \mathcal{I}_{p}^{m}\left(\mathbf{x}_{p}(t)\right)}\left|x_{i, p}(t)-\underline{x}_{p}(t)\right|,
\end{aligned}
$$

where the sets $\mathcal{I}_{p}^{M}\left(\mathbf{x}_{p}(t)\right), \mathcal{I}_{p}^{m}\left(\mathbf{x}_{p}(t)\right)$ are

$$
\begin{aligned}
& \mathcal{I}_{p}^{M}\left(\mathbf{x}_{p}(t)\right)=\left\{i \in \mathcal{V}_{f \ell}: x_{i, p}=\max _{j \in \mathcal{V}_{f \ell}} x_{j, p}(t)\right\}, \\
& \mathcal{I}_{p}^{m}\left(\mathbf{x}_{p}(t)\right)=\left\{i \in \mathcal{V}_{f \ell}: x_{i, p}=\min _{j \in \mathcal{V}_{f \ell}} x_{j, p}(t)\right\} .
\end{aligned}
$$

Furthermore, we define the $p$-th component of the hypercube $\mathcal{C}\left(\mathbf{x}^{\ell}(t)\right)$ as

$$
\mathcal{C}_{p}\left(\mathbf{x}_{p}(t)\right)=\left\{y \in \mathbb{R}: y \in\left[\bar{x}_{p}(t), \underline{x}_{p}(t)\right]\right\} .
$$

Then, in order to obtain a characterization of the generalized time derivative $d / d t\left(V_{p}\left(\mathbf{x}_{p}(t)\right)\right)$ we first analyze the term $V_{p}^{M}\left(\mathbf{x}_{p}(t)\right)$ considering two cases: i) $V_{p}^{M}\left(\mathbf{x}_{p}(t)\right)>0$, i.e., the case when there exists at least a follower outside the containment region $\mathcal{C}_{p}\left(\mathbf{x}_{p}(t)\right)$, and ii) $V_{p}^{M}\left(\mathbf{x}_{p}(t)\right)=0$, i.e., the case when the followers are all confined in the containment area $\mathcal{C}_{p}\left(\mathbf{x}_{p}(t)\right)$.

We invoke the results of Lemma 1 with $\mathcal{V}_{1}=\mathcal{V}_{f}, \mathcal{V}_{2}=\mathcal{V}_{\ell}$, and $\mathcal{V}_{3}=\mathcal{V}_{a}$ to prove that in case i) the generalized time derivative $d / d t\left(V_{p}^{M}\left(\mathbf{x}_{p}(t)\right)\right)$ is bounded by

$$
\frac{d}{d t}\left(V_{p}^{M}\left(\mathbf{x}_{p}(t)\right)\right) \leq-\left(\alpha-u_{\max }^{\ell}\right)\left|\left(\mathcal{V}_{f}, \mathcal{V}_{\ell}\right)\right|+\alpha\left|\left(\mathcal{V}_{f}, \mathcal{V}_{a}\right)\right|
$$

where $\left|\left(\mathcal{V}_{f}, \mathcal{V}_{\ell}\right)\right|$ is the cardinality of the generalized cut set introduced in Definition 3 .

We then analyze case ii) and prove that the generalized time derivative $d / d t\left(V_{p}^{M}\left(\mathbf{x}_{p}(t)\right)\right)$ is equal to zero.

We apply the same reasoning to the term $V_{p}^{m}\left(\mathbf{x}_{p}(t)\right)$ and then collectively stacks the bounds on the terms $V_{p}^{M}\left(\mathbf{x}_{p}(t)\right)$ and $V_{p}^{m}\left(\mathbf{x}_{p}(t)\right)$ to obtain the following bound on the generalized time derivative $d / d t\left(V_{p}\left(\mathbf{x}_{p}(t)\right)\right)$ as

$$
\frac{d}{d t}\left(V_{p}\left(\mathbf{x}_{p}(t)\right)\right) \leq-2\left(\alpha-u_{\max }^{\ell}\right)\left|\left(\mathcal{V}_{f}, \mathcal{V}_{\ell}\right)\right|+2 \alpha\left|\left(\mathcal{V}_{f}, \mathcal{V}_{a}\right)\right|
$$

when there exists at least a follower outside the containment region $\mathcal{C}_{p}\left(\mathbf{x}_{p}(t)\right)$.

Furthermore, we prove that the generalized time derivative $d / d t\left(V_{p}\left(\mathbf{x}_{p}(t)\right)\right)$ is equal to zero when the followers are all contained in the area delimited by the position of the leaders $\mathcal{C}_{p}\left(\mathbf{x}_{p}(t)\right)$.

We resort to Theorem 2 (Finite-Time Stability) to conclude that the followers reach in finite-time $T$ the containment area $\mathcal{C}_{p}\left(\mathbf{x}_{p}(t)\right)$ and then remain confined in it $\forall t \geq T$.

Finally, we show that the result follows applying the same reasoning to all the $d$ components of the state.

From the result above it follows that for any leader control input $u_{i}^{\ell}(t), \forall i \in \mathcal{V}_{\ell}$, upper bounded by $u_{\max }^{\ell}$, there will always exists a positive gain $\alpha \gg u_{\max }^{\ell}$ such that the condition required in eq. 20, becomes

$$
\left(r-n_{a}\right) \min \left\{n_{f}, n_{\ell}, s\right\}>\left|\left(\mathcal{V}_{f}, \mathcal{V}_{a}\right)\right| .
$$

To further analyze eq. 20 and obtain a clear condition on the maximum number of adversarial agents for which the network is resilient, we note that it holds

$$
n_{a}<r-\frac{\left|\left(\mathcal{V}_{f}, \mathcal{V}_{a}\right)\right|}{\left(1-\frac{u_{\max }^{\ell}}{\alpha}\right) \min \left\{n_{f}, n_{\ell}, s\right\}} .
$$

We now characterize the convergence time of the considered control strategy described in Theorem 3

Theorem 4. Consider a multi-robot system such that Theorem 3 holds. Then, the containment area $\mathcal{C}\left(\mathrm{x}^{\ell}(t)\right)$ defined as eq. (13) is reached in a finite-time $T$ that is upper bounded by a finite positive value $\bar{T}$ equal to

$$
\bar{T}=\frac{1}{\beta} \max _{p \in\{1, \ldots, d\}}\left\{\max \left\{V_{p}^{M}\left(\mathbf{x}_{p}(0)\right), V_{p}^{m}\left(\mathbf{x}_{p}(0)\right)\right\}\right\},
$$

where $\beta$ is defined as

$$
\beta=-\left(\alpha-u_{\max }^{\ell}\right)\left|\left(\mathcal{V}_{f}, \mathcal{V}_{\ell}\right)\right|+\alpha\left|\left(\mathcal{V}_{f}, \mathcal{V}_{a}\right)\right|,
$$

and the terms $V_{p}^{M}\left(\mathbf{x}_{p}(0)\right), V_{p}^{m}\left(\mathbf{x}_{p}(0)\right)$ are

$$
\begin{aligned}
V_{p}^{M}\left(\mathbf{x}_{p}(0)\right) & =\sum_{i \in \mathcal{I}_{p}^{M}\left(\mathbf{x}_{p}(0)\right)}\left|x_{i, p}(0)-\bar{x}_{p}(0)\right|, \\
V_{p}^{m}\left(\mathbf{x}_{p}(0)\right) & =\sum_{i \in \mathcal{I}_{p}^{m}\left(\mathbf{x}_{p}(0)\right)}\left|x_{i, p}(0)-\underline{x}_{p}(0)\right| .
\end{aligned}
$$

Proof. In order to establish the convergence time let us compute the following inequality

$$
\begin{aligned}
V_{p}^{h}\left(\mathbf{x}_{p}(t)\right) & =V_{p}^{h}\left(\mathbf{x}_{p}(0)\right)+\int_{0}^{t} \frac{d}{d t}\left(V_{p}^{h}(x(\tau))\right) \mathrm{d} \tau \\
& \leq V_{p}^{h}(\mathbf{x}(0))-\int_{0}^{t} \beta \mathrm{d} \tau=V_{p}^{h}\left(\mathbf{x}_{p}(0)\right)-\beta t,
\end{aligned}
$$

where $h \in\{m, M\}$. An upper bound on the convergence time $T$ is then

$$
T_{p}=\frac{1}{\beta} \max \left\{V_{p}^{M}\left(\mathbf{x}_{p}(0)\right), V_{p}^{m}\left(\mathbf{x}_{p}(0)\right)\right\} .
$$

Since the evolution of the multi-robot system is decoupled along its $d$ dimensions we can conclude that the followers 
will reach the containment region $\mathcal{C}\left(\mathrm{x}^{\ell}(t)\right)$ with convergence time $T \leq \max _{p \in\{1, \ldots, d\}} T_{p}$. The result thus follows.

To conclude, we extend the results of Theorem 3 to consider the case of a time-varying interaction graph $\mathcal{G}(t)$.

Theorem 5. Consider a multi-robot system consisting of $n_{f}$ followers, $n_{\ell}$ leaders, and $n_{a}$ adversarial agents and with network topology encoded by an undirected time-varying graph $\mathcal{G}(t)=(\mathcal{V}, \mathcal{E}(t))$. Let the dynamics of the robots be described by eqs. (10), (11), and (12) under the assumption of locally essentially boundedness of their control inputs. Moreover, let the control inputs of the leaders be bounded by $u_{\max }^{\ell} \in \mathbb{R}_{\geq 0}$, i.e., $\left\|u_{i}^{\ell}(t)\right\|_{\infty} \leq u_{\max }^{\ell}$. Let the followers execute the control protocol in eq. (17) and let $\Omega$ be a set of instants of time of measure zero. If

- the edge set $\mathcal{E}(t)$ changes only at instants $t \in \Omega$;

- the conditions in Theorem 3 hold for all $t \notin \Omega$.

then, there exists a finite-time $T>0$ such that

$$
x_{i}(t) \in \mathcal{C}\left(\mathbf{x}^{\ell}(t)\right), \quad \forall i \in \mathcal{V}_{f}, \quad \forall t \geq T,
$$

where the containment region $\mathcal{C}\left(\mathrm{x}^{\ell}(t)\right)$ is defined as in eq. 13 and the finite-time $T$ is upper bounded by $\bar{T}$ defined as in eq. 30.

Proof. The proof is reported in Appendix B

\section{NUMERICAL SIMULATIONS}

In this section, we provide numerical results to prove the effectiveness of our theoretical findings. In particular, we consider a multi-robot system composed of 44 robotic agents moving in a 2 dimensional $(13,8)$-robust time-invariant network. The agents are partitioned as follows: 32 followers, 8 leaders, and 4 adversarial agents.

The leaders evolve using the following control inputs

$$
\begin{array}{rlrl}
u_{i}^{\ell}(t) & =\left[\begin{array}{ll}
-\frac{1}{4} & \frac{1}{8}
\end{array}\right]^{T}, & i \in\{33,34,36,39\}, \\
u_{i}^{\ell}(t)=\left[\begin{array}{ll}
-\frac{1}{4} & \frac{1}{4}
\end{array}\right]^{T}, & i \in\{38\}, \\
u_{i}^{\ell}(t)=\left[\begin{array}{ll}
-\frac{1}{8} & \frac{1}{4}
\end{array}\right]^{T}, & i \in\{40\}, \\
u_{i}^{\ell}(t)=\sin (2 t)\left[\begin{array}{ll}
\frac{1}{4} & \frac{1}{2}
\end{array}\right]^{T}, & i \in\{35\}, \\
u_{i}^{\ell}(t)=\cos (t)\left[\begin{array}{ll}
-\frac{1}{4} & \frac{1}{2}
\end{array}\right]^{T}, & i \in\{37\} .
\end{array}
$$

Since the maximum speed of the leaders is $u_{\max }^{\ell}=\frac{1}{2}$, the followers execute protocol (17) with gain $\alpha=10$, and the number of connections between followers and adversarial agents is 65 , it follows that the conditions of Theorem 3 are satisfied, allowing the multi-robot system to solve in finitetime the containment problem introduced in Problem 1

The adversarial robots apply the following control inputs

$$
\begin{array}{ll}
u_{i}^{a}(t)=\left[\begin{array}{ll}
\frac{3}{5} & \frac{3}{2} \sin (t)+\frac{3}{20} t
\end{array}\right]^{T}, \quad i \in\{41,42\}, \\
u_{i}^{a}(t)=\left[\begin{array}{ll}
\frac{1}{2} & 2 \cos (2 t)+\frac{1}{10} t
\end{array}\right]^{T}, \quad i \in\{43,44\} .
\end{array}
$$

In Figure 3 the results of the numerical simulation are reported. In particular, in Fig. (3a) it is depicted the starting position of the robots, whereas in Fig. (3b) the final one is shown. The followers are depicted with red circles, the leaders with green squares, and the adversarial agents with blue triangles. As shown in Fig. (3b), the followers are able to reach and remain contained in the hypercube defined by the positions of the leaders. The adversarial agents are not shown in the figure since they moved far away from the containment region $\mathcal{C}\left(\mathbf{x}^{\ell}(t)\right)$, whereas the followers have reached consensus and are all overlapped near leader 33. For the sake of readability the agents' connection are not shown. Figs. (3c) and (3d) shows the agents' evolution along the $\mathbf{x}_{1}$ and $\mathbf{x}_{2}$ axes, respectively. The evolution of the states $x_{i, 1}$ and $x_{i, 2}$ are depicted with dashed colored lines with respect to the type of agent they correspond to, whereas the containment limits $\bar{x}_{1}, \underline{x}_{1}, \bar{x}_{2}$, and $\underline{x}_{2}$ are depicted with solid purple lines. In both cases the agents are able to reach consensus and, despite being influenced by the evolution of the adversarial robots, manage to remain and track the movement of the hypercube $\mathcal{C}\left(\mathbf{x}^{\ell}(t)\right)$.

We reiterate that in our setting the robots are not aware of the role of other agents in the network.

\section{Conclusions}

In this paper the resilient containment control problem in a multi-robot system with the presence of anonymous adversarial agents was addressed. Under the assumption of neighbors detection and the presence of $d-1$ common bearing directions, we characterized topological conditions that enabled a local interaction protocol based on the sign function to be robust against adversarial agents with arbitrary control inputs. In particular, under such topological conditions, we proved that the dynamic containment problem can be solved if the velocity of the leaders is bounded and the gains of the followers are at least equal to the speed of the leaders. Furthermore, we proved that in the case of timevarying graphs, the resilient containment control problem can still be achieved under the same topological conditions. We concluded the paper showing numerical results with a team composed of 32 followers, 8 leaders, and 4 adversarial agents in a 2 dimensional environment, proving the effectiveness of the proposed control strategy. Future work will investigate multi-robot system with higher system dynamics while also removing the hypothesis of common bearing directions.

\section{APPENDIX A \\ ProOF OF THEOREM 3}

In order to prove the result we are first going to detail the behaviour of the system considering a single $p$-th component; then we will extend this reasoning to any $d$ dimensional space.

Let us consider now the sets $\mathcal{I}_{p}^{M}\left(\mathbf{x}_{p}(t)\right)$ and $\mathcal{I}_{p}^{m}\left(\mathbf{x}_{p}(t)\right)$ collecting followers and leaders with the maximum and minimum value in the network with respect to the $p$-th component defined as

$$
\begin{aligned}
& \mathcal{I}_{p}^{M}\left(\mathbf{x}_{p}(t)\right)=\left\{i \in \mathcal{V}_{f \ell}: x_{i, p}=\max _{j \in \mathcal{V}_{f \ell}} x_{j, p}(t)\right\}, \\
& \mathcal{I}_{p}^{m}\left(\mathbf{x}_{p}(t)\right)=\left\{i \in \mathcal{V}_{f \ell}: x_{i, p}=\min _{j \in \mathcal{V}_{f \ell}} x_{j, p}(t)\right\} .
\end{aligned}
$$

Next, let us introduce the following non-smooth Lyapunov function $V_{p}\left(\mathbf{x}_{p}(t)\right): \mathbb{R}^{n_{f}+n_{\ell}} \rightarrow \mathbb{R}_{\geq 0}$ defined as

$$
V_{p}\left(\mathbf{x}_{p}(t)\right)=V_{p}^{M}\left(\mathbf{x}_{p}(t)\right)+V_{p}^{m}\left(\mathbf{x}_{p}(t)\right),
$$




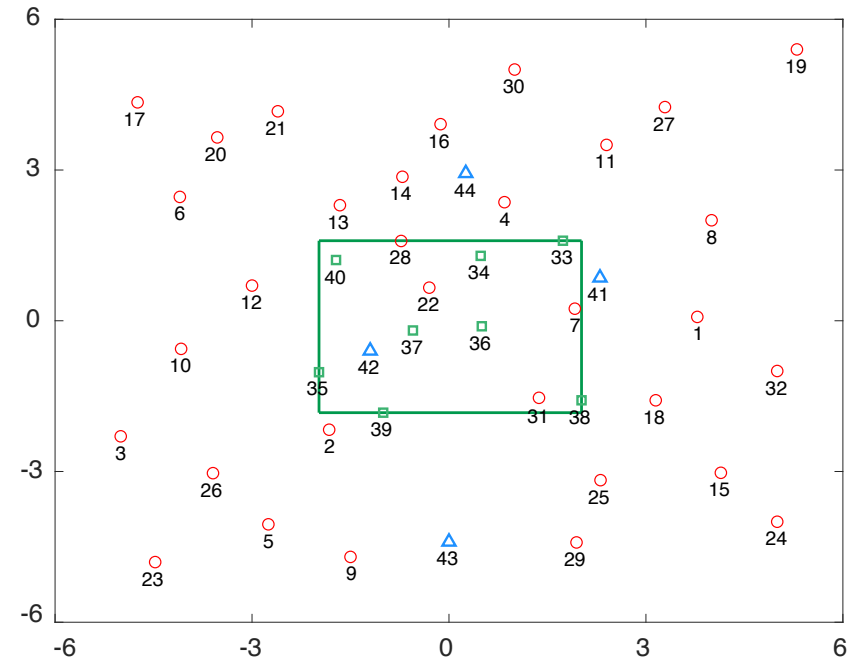

(a) Position of the team of robots at initial time $t=0 \mathrm{~s}$.

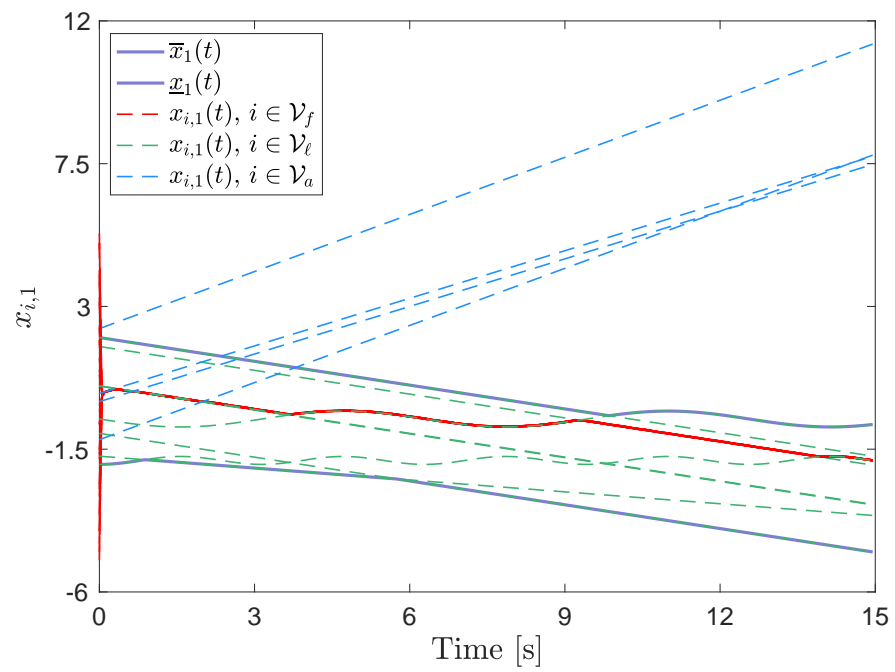

(c) Evolution of the first component $x_{i, 1}(t)$ of the robots with respect to the(d) Evolution of the second component $x_{i, 2}(t)$ of the robots with respect to limits $\bar{x}_{1}(t)$ and $\underline{x}_{1}(t)$ of the containment section $\mathcal{C}_{1}(t)$.

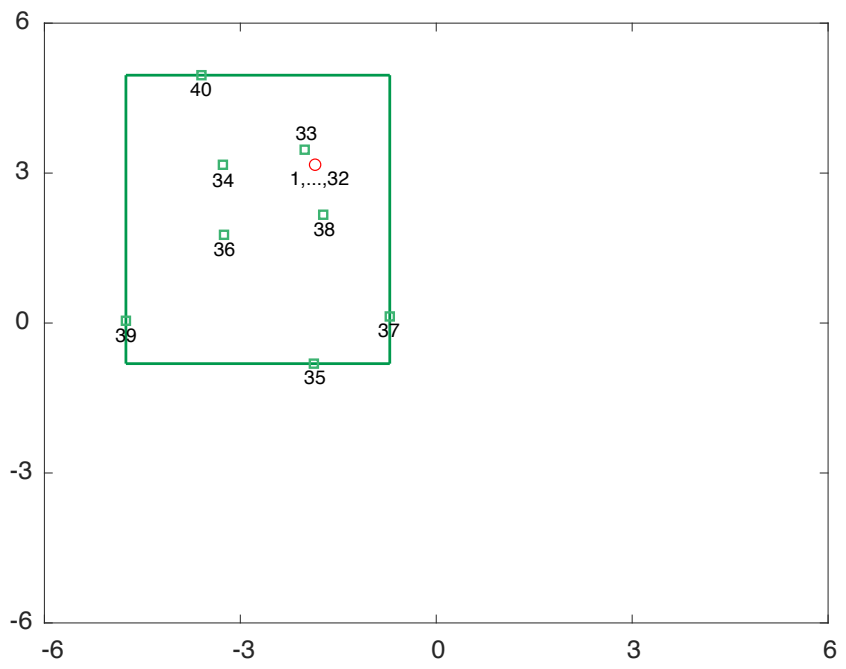

(b) Position of the team of robots at final time $t=15 \mathrm{~s}$.

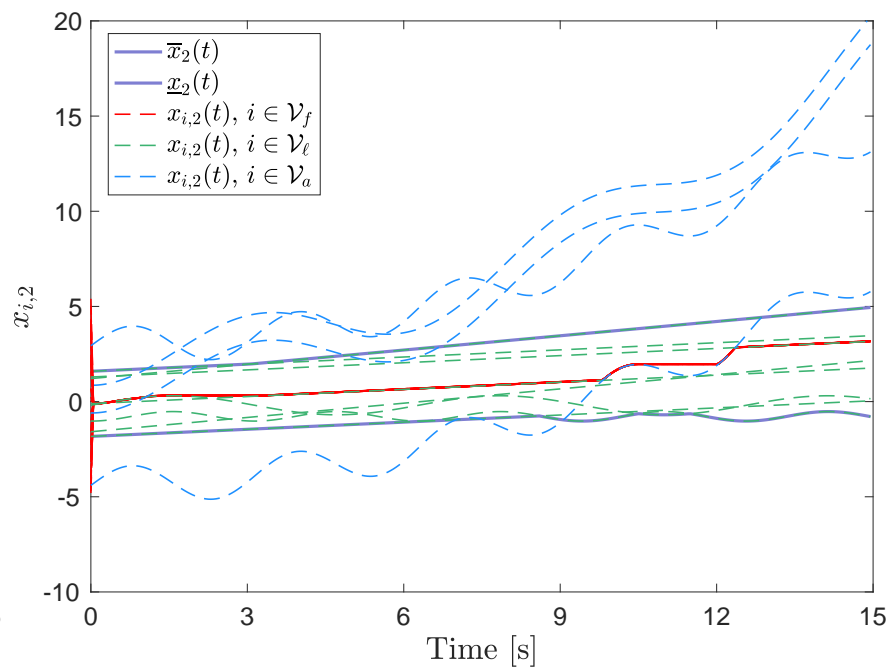

the limits $\bar{x}_{2}(t)$ and $\underline{x}_{2}(t)$ of the containment section $\mathcal{C}_{2}(t)$.

Figure 3. A multi-robot system composed of 32 followers, 8 leaders, and 4 adversarial agents in a $(13,8)$-robust network.

composed by the terms $V_{p}^{M}\left(\mathbf{x}_{p}(t)\right)$ and $V_{p}^{m}\left(\mathbf{x}_{p}(t)\right)$

$$
\begin{aligned}
V_{p}^{M}\left(\mathbf{x}_{p}(t)\right) & =\sum_{i \in \mathcal{I}_{p}^{M}\left(\mathbf{x}_{p}(t)\right)} V_{i, p}^{M}\left(\mathbf{x}_{p}(t)\right) \\
V_{p}^{m}\left(\mathbf{x}_{p}(t)\right) & =\sum_{i \in \mathcal{I}_{p}^{m}\left(\mathbf{x}_{p}(t)\right)} V_{i, p}^{m}\left(\mathbf{x}_{p}(t)\right)
\end{aligned}
$$

where the variables $V_{i, p}^{M}\left(\mathbf{x}_{p}(t)\right)$ and $V_{i, p}^{m}\left(\mathbf{x}_{p}(t)\right)$ are

$$
\begin{gathered}
V_{i, p}^{M}\left(\mathbf{x}_{p}(t)\right)=\left|x_{i, p}(t)-\bar{x}_{p}(t)\right| \\
V_{i, p}^{m}\left(\mathbf{x}_{p}(t)\right)=\left|x_{i, p}(t)-\underline{x}_{p}(t)\right| .
\end{gathered}
$$

Moreover, let us also introduce the $p$-th component of the hypercube $\mathcal{C}\left(\mathbf{x}^{\ell}(t)\right)$ as

$$
\mathcal{C}_{p}\left(\mathbf{x}_{p}(t)\right)=\left\{y \in \mathbb{R}: y \in\left[\bar{x}_{p}(t), \underline{x}_{p}(t)\right]\right\} .
$$

It should be noticed that in virtue of the previous definitions, the following holds

$$
V_{p}\left(\mathbf{x}_{p}(t)\right)>0 \Longleftrightarrow\left\{\begin{array}{l}
\exists i \in \mathcal{I}_{p}^{M}\left(\mathbf{x}_{p}(t)\right): x_{i, p}(t) \notin \mathcal{C}_{p}\left(\mathbf{x}_{p}(t)\right) \\
\quad \text { or } \\
\exists i \in \mathcal{I}_{p}^{m}\left(\mathbf{x}_{p}(t)\right): x_{i, p}(t) \notin \mathcal{C}_{p}\left(\mathbf{x}_{p}(t)\right)
\end{array}\right.
$$

that is, the Lyapunov function in eq. (40) is positive as long as there exists an agent in the maximum or minimum sets $\mathcal{I}_{p}^{M}\left(\mathbf{x}_{p}(t)\right), \mathcal{I}_{p}^{m}\left(\mathbf{x}_{p}(t)\right)$ that is not inside in the containment region $\mathcal{C}_{p}\left(\mathbf{x}_{p}(t)\right)$.

From now on, dependence on time $t$ and state $\mathbf{x}$ will be used only when introducing new concepts or variables and omitted otherwise for the sake of clarity.

In the following, we will analyze only the first term of eq. 40 , i.e., $V_{p}^{M}$, since a similar analysis can be conducted on the term $V_{p}^{m}$ adjusting some details that will be discussed later. 
Since $V_{p}^{M}$ is a Lipschitz regular function we can resort to the chain rule given in Theorem 1 to compute the set-valued Lie derivative $\tilde{V}_{p}^{M}\left(\mathbf{x}_{p}\right)$ as

$$
\dot{\tilde{V}}_{p}^{M}\left(\mathbf{x}_{p}\right)=\bigcap_{\xi_{p} \in \partial V_{p}^{M}\left(\mathbf{x}_{p}\right)} \xi_{p}^{T} K[f]\left(\mathbf{x}_{p}\right),
$$

where the structure of the generalized gradient $\partial V_{p}^{M}\left(\mathbf{x}_{p}\right)$ and the set-valued map $K[f]\left(\mathbf{x}_{p}\right)$ are now going to be characterized.

In order to describe the form of the generalized gradient $\partial V_{p}^{M}\left(\mathbf{x}_{p}\right)$, let us introduce the set $\mathscr{L}\left(\mathbf{x}_{p}(t)\right)$ denoting the leaders possessing the maximum value $\bar{x}_{p}(t)$ as

$$
\mathscr{L}\left(\mathbf{x}_{p}(t)\right)=\left\{i \in \mathcal{V}_{\ell}: x_{i, p}(t)=\bar{x}_{p}(t)\right\}=\left\{l_{1}, \ldots, l_{L}\right\},
$$

where $\left\{l_{1}, \ldots, l_{L}\right\}$ denotes the elements of the set $\mathscr{L}\left(\mathbf{x}_{p}\right)$. Exploiting [44, Proposition 2.3.12] and [44, Proposition 2.3.3], the generalized gradient assumes the following form

$$
\begin{aligned}
\partial V_{p}^{M} & \subseteq \operatorname{co}\left\{\partial V_{p}^{M, l}\left(\mathbf{x}_{p}\right): l \in \mathscr{L}\left(\mathbf{x}_{p}\right)\right\} \\
& \subseteq \operatorname{co}\left\{\sum_{i \in \mathcal{I}_{p}^{M}} \partial V_{i, p}^{M, l}: l \in \mathscr{L}\left(\mathbf{x}_{p}\right)\right\},
\end{aligned}
$$

where the elements $\partial V_{i, p}^{M, l}\left(\mathbf{x}_{p}\right)$ are defined as

$$
\partial V_{i, p}^{M, l}\left(\mathbf{x}_{p}\right)=\left[0, \ldots, 0, \underset{i}{i \text {-th }}, \underset{l \text {-th }}{0, \ldots, 0,-S_{i}, 0, \ldots, 0}\right]^{T},
$$

with $S_{i}=\operatorname{SIGN}\left(x_{i, p}-\bar{x}_{p}\right)$.

Furthermore, the generalized gradient $\partial V_{p}^{M, l}$ satisfies

$$
\begin{gathered}
\partial V_{p}^{M, l} \subseteq \sum_{i \in \mathcal{I}_{p}^{M}} \partial V_{i, p}^{M, l}\left(\mathbf{x}_{p}\right)= \\
{\left[0, \ldots, S_{\nu_{1}}, \ldots, S_{\nu_{q}}, \ldots,-\sum_{\substack{i \in \mathcal{I}_{p}^{M} \\
l \text {-th }}} S_{i}, \ldots, S_{\nu_{h}}, \ldots, 0\right]^{T}} \\
\nu_{1} \text {-th } \quad \nu_{q_{h} \text {-th }}
\end{gathered}
$$

where the indices $\left\{\nu_{1}, \ldots, \nu_{q}, \ldots, \nu_{h}\right\}$ correspond to the agents in the set $\mathcal{I}_{p}^{M}$. Note that the $l$-th element is in that position because the leaders can also be part of the set $\mathcal{I}_{p}^{M}$.

The structure of the convex hull is then the following

$$
\begin{aligned}
& \operatorname{co}\left\{\sum_{i \in \mathcal{I}_{p}^{M}} \partial V_{i, p}^{M, l}: l \in \mathscr{L}\left(\mathbf{x}_{p}\right)\right\}= \\
& \operatorname{co}\left\{\left[0, \ldots, S_{\nu_{1}}, \ldots, S_{\nu_{q}}, \ldots,-\sum_{i \in \mathcal{I}_{p}^{M}} S_{i}, \ldots, S_{\nu_{h}}, \ldots, 0\right]^{T},\right. \\
& \nu_{\nu_{1} \text {-th }} \quad \nu_{q} \text {-th } \quad \nu_{l_{h} \text {-th }},
\end{aligned}
$$

$$
\begin{aligned}
& \left.\ldots,\left[0, \ldots, S_{\nu_{1}}, \ldots, S_{\nu_{q}}, \ldots, S_{\nu_{w}}, \ldots,-\sum_{i \in \mathcal{I}_{p}^{M}} S_{i}, \ldots, 0\right]^{T}\right\} \\
& \nu_{1} \text {-th } \quad \nu_{q} \text {-th } \quad \nu_{w} \text {-th } \quad l_{L} \text {-th } \\
& =\left[0, \ldots, S_{\nu_{1}}, \ldots, S_{\nu_{h}}, \ldots,-\gamma_{l_{1}} \sum_{i \in \mathcal{I}_{p}^{M}} S_{i}+\sum_{l \in \mathscr{L} \backslash\left\{l_{1}\right\}} \gamma_{l} S_{l_{1}}\right. \text {, } \\
& \left.\ldots,-\gamma_{l_{L}} \sum_{i \in \mathcal{I}_{p}^{M}} S_{i}+\sum_{l \in \mathscr{L} \backslash\left\{l_{L}\right\}} \gamma_{l} S_{l_{L}}, \ldots, 0\right]^{T},
\end{aligned}
$$

where $\gamma_{l} \in[0,1], \forall l \in \mathscr{L}$ and are such that $\sum_{l \in \mathscr{L}} \gamma_{l}=1$. The zero elements in eq. (52) correspond to the followers and leaders that are not part of the set $\mathcal{I}_{p}^{M}$ and $\mathscr{L}$, respectively. For the sake of brevity in the following we are going to denote eq. 52 as $\operatorname{co}\left(\partial V_{p}^{M}\right)$.

The structure of the set-valued map $K[f]\left(\mathbf{x}_{p}\right)$ is the following

$$
K[f]\left(\mathbf{x}_{p}\right) \subseteq\left[K\left[f_{1}\right]\left(\mathbf{x}_{p}\right), \ldots, K\left[f_{n_{f}+n_{\ell}}\right]\left(\mathbf{x}_{p}\right)\right]^{T},
$$

where terms $K\left[f_{i}\right]\left(\mathbf{x}_{p}\right)$ are defined in virtue of the agents dynamics in eqs. (10), (11) and (17) as

$$
\begin{aligned}
K\left[f_{i}\right]\left(\mathbf{x}_{p}\right) & =K\left[-\alpha \sum_{j \in \mathcal{N}_{i}} \operatorname{sign}\left(x_{i, p}-x_{j, p}\right)\right] \\
& \subseteq-\alpha \sum_{j \in \mathcal{N}_{i}} K\left[\operatorname{sign}\left(x_{i, p}-x_{j, p}\right)\right] \\
& =-\alpha \sum_{j \in \mathcal{N}_{i}} \operatorname{SIGN}\left(x_{i, p}-x_{j, p}\right), \quad i \in \mathcal{V}_{f},
\end{aligned}
$$

if the agent $i$ is a follower, or as

$$
K\left[f_{i}\right]\left(\mathbf{x}_{p}\right)=\left\{u_{i, p}^{\ell}\right\}, \quad i \in \mathcal{V}_{\ell},
$$

if the agent $i$ is a leader with $u_{i, p}^{\ell} \in \mathbb{R}$ the $p$-th component of its control input $u_{i}^{\ell}$.

Consider now a generic vector $\bar{\xi}_{p} \in \operatorname{co}\left(\partial V_{p}^{M}\right)$. We want to analyze the dot product $\bar{\xi}_{p}^{T} K[f]\left(\mathbf{x}_{p}\right)$ in order to derive properties that will hold also for the elements $\xi_{p} \in \partial V_{p}^{M}$ required to compute the set-valued Lie derivative $\dot{\tilde{V}}_{p}^{M}$ introduced in eq. 477 . Denoting for convenience the vector $\bar{\xi}_{p}$ as $\bar{\xi}_{p}=\left[\bar{\xi}_{1, p}, \ldots, \bar{\xi}_{n_{f}+n_{\ell}, p}\right]^{T}$, it holds from eq. 53 that

$$
\bar{\xi}_{p}^{T} K[f]\left(\mathbf{x}_{p}\right) \subseteq \sum_{i=1}^{n_{f}+n_{\ell}} \bar{\xi}_{i, p} K\left[f_{i}\right]\left(\mathbf{x}_{p}\right) .
$$

Two different cases are now considered:

- Case i) $V_{p}^{M}>0$;

- Case ii) $V_{p}^{M}=0$.

From the definition of the set $\mathcal{I}_{p}^{M}$ in eq. 38, , it follows that in the first case there exists at least a follower $i \in \mathcal{I}_{p}^{M}$ such that $x_{i, p}>\bar{x}_{p}$ whereas in the latter all the followers are inside the containment area $\mathcal{C}_{p}$.

Case i). In this case the set $\mathcal{I}_{p}^{M}$ is composed by followers $\mathcal{V}_{f}$ only, otherwise $V_{p}^{M}$ would have been equal to 
zero. We can therefore establish the value of the set-valued function $\operatorname{SIGN}\left(x_{i, p}-\bar{x}_{p}\right)$ as

$$
\operatorname{SIGN}\left(x_{i, p}-\bar{x}_{p}\right)=\{1\}, \quad \forall i \in \mathcal{I}_{p}^{M},
$$

obtaining the following form for the convex hull $\operatorname{co}\left(\partial V_{p}^{M}\right)$

$$
\begin{aligned}
& \operatorname{co}\left(\partial V_{p}^{M}\right)= \\
& {\left[0, \ldots, 1, \ldots, 1, \ldots,-\gamma_{l_{1}}\left|\mathcal{I}_{p}^{M}\right|, \ldots,-\gamma_{l_{L}}\left|\mathcal{I}_{p}^{M}\right|, \ldots, 0\right]^{T} .} \\
& \nu_{1} \text {-th } \nu_{h} \text {-th }
\end{aligned}
$$

Notice that the terms $\sum_{l \in \mathscr{L} \backslash\left\{l_{w}\right\}} \gamma_{l} S_{l_{w}}$ are not present because the leaders are not members of $\mathcal{I}_{p}^{M}$. We also want to point out that in this case the generalized gradient is a set which contains a single point, i.e., a singleton.

In virtue of eqs. (54), 55) and (58) we can further develop eq. (56) as follows

$$
\begin{aligned}
& \sum_{i=1}^{n_{f}+n_{\ell}} \bar{\xi}_{i, p} K\left[f_{i}\right]\left(\mathbf{x}_{p}\right) \\
= & \sum_{i \in \mathcal{I}_{p}^{M}}\left(-\alpha \sum_{j \in \mathcal{N}_{i}} \operatorname{SIGN}\left(x_{i, p}-x_{j, p}\right)\right)-\left|\mathcal{I}_{p}^{M}\right| \sum_{l \in \mathscr{L}} \gamma_{l} u_{l, p}^{\ell} \\
= & \sum_{i \in \mathcal{I}_{p}^{M}}\left(-\alpha \sum_{j \in \mathcal{N}_{i}} \operatorname{SIGN}\left(x_{i, p}-x_{j, p}\right)-\sum_{l \in \mathscr{L}} \gamma_{l} u_{l, p}^{\ell}\right) .
\end{aligned}
$$

We are going now to detail the elements that compose eq. (59) discerning the contributions of the neighbors $\mathcal{N}_{i}$ with respect to the kind of the neighbor agent, i.e., if they are followers $\mathcal{V}_{f}$, leaders $\mathcal{V}_{\ell}$, or adversarial agents $\mathcal{V}_{a}$. The following holds

$$
\begin{aligned}
& -\alpha \sum_{j \in \mathcal{N}_{i}} \operatorname{SIGN}\left(x_{i, p}-x_{j, p}\right)=-\alpha \sum_{j \in \mathcal{N}_{i} \cap \mathcal{V}_{f}} \operatorname{SIGN}\left(x_{i, p}-x_{j, p}\right) \\
& -\alpha \sum_{j \in \mathcal{N}_{i} \cap \mathcal{V}_{\ell}} \operatorname{SIGN}\left(x_{i, p}-x_{j, p}\right)-\alpha \sum_{j \in \mathcal{N}_{i} \cap \mathcal{V}_{a}} \operatorname{SIGN}\left(x_{i, p}-x_{j, p}\right) .
\end{aligned}
$$

Moreover, we can discern the contributions in two types: the ones that come from neighbors that are at consensus with the agent $i$ and the ones that come from the rest of the agents, that is

$$
\begin{aligned}
& -\alpha \sum_{j \in \mathcal{N}_{i}} \operatorname{SIGN}\left(x_{i, p}-x_{j, p}\right)=-\alpha \sum_{\substack{j \in \mathcal{N}_{i} \cap \mathcal{V}_{f} \\
j \notin \mathcal{I}_{p}^{M}}} \operatorname{SIGN}\left(x_{i, p}-x_{j, p}\right) \\
& -\alpha \sum_{\substack{j \in \mathcal{N}_{i} \cap \mathcal{V}_{f} \\
j \in \mathcal{I}_{p}^{M}}} \operatorname{SIGN}\left(x_{i, p}-x_{j, p}\right)-\alpha \sum_{\substack{j \in \mathcal{N}_{i} \cap \mathcal{V}_{\ell} \\
j \notin \mathcal{I}_{p}^{M}}} \operatorname{SIGN}\left(x_{i, p}-x_{j, p}\right) \\
& -\alpha \sum_{\substack{j \in \mathcal{N}_{i} \cap \mathcal{V}_{\ell} \\
j \in \mathcal{I}_{p}^{M}}} \operatorname{SIGN}\left(x_{i, p}-x_{j, p}\right)-\alpha \sum_{\substack{j \in \mathcal{N}_{i} \cap \mathcal{V}_{a}\\
}} \operatorname{SIGN}\left(x_{i, p}-x_{j, p}\right) .
\end{aligned}
$$

To further develop eq. (59) we now point out a few observations. First of all, since the graph $\mathcal{G}$ is undirected, the followers $x_{i, p} \in \mathcal{I}_{p}^{M}$ mutually cancel their contributions, i.e.,

$$
\sum_{i \in \mathcal{I}_{p}^{M}}\left(-\underset{\substack{j \in \mathcal{N}_{i} \cap \mathcal{V}_{f} \\ j \in \mathcal{I}_{p}^{M}}}{ } \operatorname{SIGN}\left(x_{i, p}-x_{j, p}\right)\right)=0 .
$$

Second, as already mentioned above, the set $\mathcal{I}_{p}^{M}$ is composed only of followers $\mathcal{V}_{f}$ when $V_{p}^{M}>0$, i.e., $\mathcal{I}_{p}^{M} \cap \mathcal{V}_{\ell}=\emptyset$. Moreover, given the definition of the set $\mathcal{I}_{p}^{M}$ in eq. 38 we can clearly define the value of the following term

$$
\operatorname{SIGN}\left(x_{i, p}-x_{j, p}\right)=\{1\} \quad \forall i \in \mathcal{I}_{p}^{M}, \forall j \in\left(\mathcal{N}_{i} \cap \mathcal{V}_{f \ell} \backslash \mathcal{I}_{p}^{M}\right)
$$

Finally, considering the worst case scenario, the adversarial neighbors $x_{j, p} \in \mathcal{V}_{a}$ interactions can be bounded as

$$
-\alpha \sum_{j \in \mathcal{N}_{i} \cap \mathcal{V}_{a}} \operatorname{SIGN}\left(x_{i, p}-x_{j, p}\right) \subseteq-\alpha \sum_{j \in \mathcal{N}_{i} \cap \mathcal{V}_{a}}[-1,1] .
$$

Putting everything together we get

$$
\begin{aligned}
& \sum_{i=1}^{n_{f}+n_{\ell}} \bar{\xi}_{i, p} K\left[f_{i}\right]\left(\mathbf{x}_{p}\right) \\
& \subseteq \sum_{i \in \mathcal{I}_{p}^{M}}\left(-\sum_{\substack{j \in \mathcal{N}_{i} \cap \mathcal{V}_{f \ell} \\
j \notin \mathcal{I}_{p}^{M}}} \alpha-\alpha \sum_{j \in \mathcal{N}_{i} \cap \mathcal{V}_{a}}[-1,1]-\sum_{l \in \mathscr{L}} \gamma_{l} u_{l, p}^{\ell}\right) .
\end{aligned}
$$

At this point, recalling that the number of edges between two subsets of nodes $\mathcal{S}_{1}$ and $\mathcal{S}_{2}$ is the cardinality of generalized cut set $\left(\mathcal{S}_{1}, \mathcal{S}_{2}\right)$, i.e., $\left|\left(\mathcal{S}_{1}, \mathcal{S}_{2}\right)\right|$, exploiting the fact that $\sum_{l \in \mathscr{L}} \gamma_{l}=1$, and bounding the leaders control inputs from above with the positive constant $u_{\text {max }}^{\ell} \in \mathbb{R}_{>0}$ such that $\left|u_{i, p}^{\ell}\right| \leq u_{\max }^{\ell}, \forall i \in \mathcal{V}_{\ell}$, we obtain the following

$$
\begin{aligned}
\dot{\tilde{V}}_{p}^{M}\left(\mathbf{x}_{p}\right) \subseteq \alpha & -\left|\left(\mathcal{V}_{f}, \mathcal{V}_{\ell}\right)\right|-\left|\left(\mathcal{V}_{f}, \mathcal{V}_{a}\right)\right|-\frac{u_{\max }^{\ell}}{\alpha}\left|\left(\mathcal{V}_{f}, \mathcal{V}_{\ell}\right)\right| \\
& \left.-\left|\left(\mathcal{V}_{f}, \mathcal{V}_{\ell}\right)\right|+\left|\left(\mathcal{V}_{f}, \mathcal{V}_{a}\right)\right|+\frac{u_{\max }^{\ell}}{\alpha}\left|\left(\mathcal{V}_{f}, \mathcal{V}_{\ell}\right)\right|\right]
\end{aligned}
$$

It then follows from eq. 66 that the set-valued Lie derivative $\dot{\tilde{V}}_{p}^{M}$ is negative definite if

$$
-\left(\alpha-u_{\max }^{\ell}\right)\left|\left(\mathcal{V}_{f}, \mathcal{V}_{\ell}\right)\right|+\alpha\left|\left(\mathcal{V}_{f}, \mathcal{V}_{a}\right)\right|<0
$$

Applying the results of Lemma 1 with $\mathcal{V}_{1}=\mathcal{V}_{f}, \mathcal{V}_{2}=\mathcal{V}_{\ell}$, and $\mathcal{V}_{3}=\mathcal{V}_{a}$ we can infer that

$$
\left|\left(\mathcal{V}_{f}, \mathcal{V}_{\ell}\right)\right| \geq\left(r-n_{a}\right) \min \left\{n_{f}, n_{\ell}, s\right\},
$$

leading to

$$
-\left(\alpha-u_{\max }^{\ell}\right)\left(r-n_{a}\right) \min \left\{n_{f}, n_{\ell}, s\right\}+\alpha\left|\left(\mathcal{V}_{f}, \mathcal{V}_{a}\right)\right|<0
$$

and thus proving the negative definiteness of the setvalued Lie derivative $\dot{\tilde{V}}_{p}^{M}\left(\mathbf{x}_{p}\right)$. At this point, recalling that the following holds true for the generalized time derivative $d / d t\left(V_{p}^{M}\left(\mathbf{x}_{p}\right)\right) \in \dot{\tilde{V}}_{p}^{M}\left(\mathbf{x}_{p}\right)$ we obtain the following bound

$$
\frac{d}{d t}\left(V_{p}^{M}\left(\mathbf{x}_{p}\right)\right) \leq-\left(\alpha-u_{\max }^{\ell}\right)\left|\left(\mathcal{V}_{f}, \mathcal{V}_{\ell}\right)\right|+\alpha\left|\left(\mathcal{V}_{f}, \mathcal{V}_{a}\right)\right|
$$

proving the negative definiteness of the generalized time derivative $d / d t\left(V_{p}^{M}\left(\mathbf{x}_{p}\right)\right)$.

Case ii). In this case, the set $\mathcal{I}_{p}^{M}$ is composed only of agents that share the same value as $\bar{x}_{p}$, that is $\forall i \in \mathcal{I}_{p}^{M}: x_{i, p}=\bar{x}_{p}$, i.e., agents that are on the maximum border of the containment 
area $\mathcal{C}_{p}$. Notice also that in this case it holds by construction that $\mathscr{L} \subseteq \mathcal{I}_{p}^{M}$ where the inequality is strict when there exists at least a follower in the set $\mathcal{I}_{p}^{M}$. form

This implies that eq. (51) will now assume the following

$$
\begin{aligned}
\partial V_{p}^{M, l} \subseteq \sum_{i \in \mathcal{I}_{p}^{M}} \partial V_{i, p}^{M, l}\left(\mathbf{x}_{p}\right)= \\
{\left[0, \ldots, \bar{S}, \ldots, \bar{S}, \ldots,-\sum_{\substack{i \in \mathcal{I}_{p}^{M} \\
l \text {-th }}} \bar{S}, \ldots, \bar{S}, \ldots, 0\right]^{T}, } \\
\nu_{1} \text {-th } \quad \nu_{h} \text {-th }
\end{aligned}
$$

where $\bar{S}=\operatorname{SIGN}\left(\bar{x}_{p}-\bar{x}_{p}\right)$ and $l_{w}$ is a generic leader in the set $\mathscr{L}$

The convex hull $\operatorname{co}\left(\partial V_{p}^{M}\right)$ introduced in eq. 52 then becomes

$$
\begin{array}{r}
\operatorname{co}\left(\partial V_{p}^{M}\right)=\left[0, \ldots, \bar{S}, \ldots, \bar{S}, \ldots,-\gamma_{l_{1}} \sum_{i \in \mathcal{I}_{p}^{M}} \bar{S}+\sum_{l \in \mathscr{L} \backslash\left\{l_{1}\right\}} \gamma_{l} \bar{S},\right. \\
\nu_{1} \text {-th } \quad \nu_{h} \text {-th } \\
\left.\ldots,-\gamma_{l_{L}} \sum_{i \in \mathcal{I}_{p}^{M}} \bar{S}+\sum_{\substack{l \in \mathscr{L} \backslash\left\{l_{L}\right\} \\
l_{L} \text {-th }}} \gamma_{l} \bar{S}, \ldots, 0\right]^{T},
\end{array}
$$

where for a generic follower $\nu_{q} \in \mathcal{I}_{p}^{M} \cap \mathcal{V}_{f}$ the contribution on the generalized gradient is a single $\bar{S}$ whereas for a generic leader $l_{w} \in \mathscr{L}$ the contribution is composed of two terms, the first coming from the contributions when $l_{w}$ is considered as the argument of the element $\bar{x}_{p}$ and the latter when other leaders are considered the argument of the value $\bar{x}_{p}$.

We want now to prove that the intersection in eq. (47) contains only the singleton $\{0\}$. To do so, we have to show first that $\{0\} \in \operatorname{co}\left(\partial V_{p}^{M}\right)$.

To this end, let us characterize the structure convex hull $\operatorname{co}\left(\partial V_{p}^{M}\right)$ in eq. 72 by analyzing a vector $\bar{\xi}_{p} \in \operatorname{co}\left(\partial V_{p}^{M}\right)$ and its components $\bar{\xi}_{i, p}$. In particular, the following holds for an element $\bar{\xi}_{i, p}$ corresponding to a follower $i \in \mathcal{I}_{p}^{M} \cap \mathcal{V}_{f}$

$$
\bar{\xi}_{i, p} \in[-1,1],
$$

which clearly contains the element $\{0\}$. Regarding the elements corresponding to the leaders $i \in \mathscr{L}$ we have

$$
\bar{\xi}_{i, p} \in\left[-\gamma_{l_{w}}\left|\mathcal{I}_{p}^{M}\right|-\sum_{l \in \mathscr{L} \backslash\left\{l_{w}\right\}} \gamma_{l}, \gamma_{l_{w}}\left|\mathcal{I}_{p}^{M}\right|+\sum_{l \in \mathscr{L} \backslash\left\{l_{w}\right\}} \gamma_{l}\right],
$$

where the index $l_{w}$ corresponds to the agent $i$. Since $\left|\mathcal{I}_{p}^{M}\right| \geq 1$ and $\sum_{l \in \mathscr{L}} \gamma_{l}=1$ we can conclude that the interval in eq. 74 contains $\{0\}$.

Recalling eq. (56) let us now analyze the following dot product

$$
\sum_{i=1}^{n_{f}+n_{\ell}} \bar{\xi}_{i, p} K\left[f_{i}\right]\left(\mathbf{x}_{p}\right)=\sum_{i \in \mathcal{I}_{p}^{M} \cap \mathcal{V}_{f}} \bar{\xi}_{i, p}\left(-\alpha \sum_{j \in \mathcal{N}_{i}} \operatorname{SIGN}\left(x_{i, p}-x_{j, p}\right)\right)
$$

$$
+\sum_{l_{w} \in \mathscr{L}}\left(-\gamma_{l_{w}} \sum_{i \in \mathcal{I}_{p}^{M}} \bar{\xi}_{i, p}+\sum_{l \in \mathscr{L} \backslash\left\{l_{w}\right\}} \gamma_{l} \bar{\xi}_{l, p}\right) u_{l_{w}, p}^{\ell} .
$$

Considering the possible variation $\left[-u_{\max }^{\ell}, u_{\max }^{\ell}\right]$ for the control inputs $u_{l_{w}, p}^{\ell}$ of the leaders $l_{w} \in \mathscr{L}$, the fact that $\operatorname{SIGN}\left(x_{i, p}-x_{j, p}\right) \subseteq[-1,1]$, and rearranging some terms then the following holds for eq. 75

$$
\begin{aligned}
& \sum_{i=1}^{n_{f}+n_{\ell}} \bar{\xi}_{i, p} K\left[f_{i}\right]\left(\mathbf{x}_{p}\right) \subseteq \sum_{l_{w} \in \mathscr{L}} u_{l_{w}, p}^{\ell}\left(\sum_{l \in \mathscr{L} \backslash\left\{l_{w}\right\}} \gamma_{l} \bar{\xi}_{l, p}\right) \\
& \quad+\sum_{i \in \mathcal{I}_{p}^{M} \cap \mathcal{V}_{f}} \bar{\xi}_{i, p}\left(-\alpha \sum_{j \in \mathcal{N}_{i}}[-1,1]+\left[-u_{\max }^{\ell}, u_{\max }^{\ell}\right]\right) \\
& \subseteq \sum_{l_{w} \in \mathscr{L}_{l_{w}, p}\left[-u_{\max }^{\ell}, u_{\max }^{\ell}\right]} \bar{\xi}_{i \in \mathcal{I}_{p}^{M} \cap \mathcal{V}_{f}} \bar{\xi}_{i, p}\left[-\alpha\left|\mathcal{N}_{i}\right|-u_{\max }^{\ell}, \alpha\left|\mathcal{N}_{i}\right|+u_{\max }^{\ell}\right]
\end{aligned}
$$

where $\sum_{l \in \mathscr{L}} \gamma_{l}=1$ has been exploited again.

In virtue of eqs. (73) to (76) and eq. (47) we can conclude that the intersection will contain only the singleton $\{0\}$, implying that

$$
\dot{\tilde{V}}_{p}^{M}\left(\mathbf{x}_{p}\right)=\{0\},
$$

and consequently proving that $d / d t\left(V_{p}^{M}\left(\mathbf{x}_{p}\right)\right)=0$ in the case $V_{p}^{M}\left(\mathbf{x}_{p}\right)=0$.

A similar analysis can be carried out for the term $V_{p}^{m}$, replacing the upper limit $\bar{x}_{p}$ and the set $\mathcal{I}_{p}^{M}$ with the equivalent lower limit $\underline{x}_{p}$ and the set $\mathcal{I}_{p}^{m}$ and adjusting the definition of the set $\mathscr{L}$ to consider the leaders that assume the value $\underline{x}_{p}$. Doing so allows us to obtain the same bound on $d / d t\left(V_{p}^{m}\left(\mathbf{x}_{p}\right)\right)$ as

$$
\frac{d}{d t}\left(V_{p}^{m}\left(\mathbf{x}_{p}\right)\right) \leq-\left(\alpha-u_{\max }^{\ell}\right)\left|\left(\mathcal{V}_{f}, \mathcal{V}_{\ell}\right)\right|+\alpha\left|\left(\mathcal{V}_{f}, \mathcal{V}_{a}\right)\right|
$$

in the case $V_{p}^{m}\left(\mathbf{x}_{p}\right)>0$ whereas $d / d t\left(V_{p}^{m}\left(\mathbf{x}_{p}\right)\right)=0$ when $V_{p}^{m}\left(\mathbf{x}_{p}\right)=0$.

Now, since the generalized time derivatives of both $V_{p}^{M}$ and $V_{p}^{m}$ are upper bounded by $-\left(\alpha-u_{\max }^{\ell}\right)\left|\left(\mathcal{V}_{f}, \mathcal{V}_{\ell}\right)\right|+\alpha\left|\left(\mathcal{V}_{f}, \mathcal{V}_{a}\right)\right|$, the following bound on the generalized time derivative $d / d t\left(V_{p}\left(\mathbf{x}_{p}\right)\right)$ can be obtained

$$
\frac{d}{d t}\left(V_{p}\left(\mathbf{x}_{p}\right)\right) \leq-2\left(\alpha-u_{\max }^{\ell}\right)\left|\left(\mathcal{V}_{f}, \mathcal{V}_{\ell}\right)\right|+2 \alpha\left|\left(\mathcal{V}_{f}, \mathcal{V}_{a}\right)\right|
$$

when there exists at least one follower that has not yet reached the containment area $\mathcal{C}_{p}$. At the same time, we can conclude that when the followers are inside the the containment region $\mathcal{C}_{p}$ the generalized time derivative $d / d t\left(V_{p}\left(\mathbf{x}_{p}\right)\right)$ is equal to zero.

At this point, resorting to Theorem 2 (Finite-Time Stability) where $\mu$ is defined as

$$
\mu=-2\left(\left(\alpha-u_{\text {max }}^{\ell}\right)\left|\left(\mathcal{V}_{f}, \mathcal{V}_{\ell}\right)\right|-\alpha\left|\left(\mathcal{V}_{f}, \mathcal{V}_{a}\right)\right|\right)>0,
$$

we can conclude that the followers $i \in \mathcal{I}_{p}^{M} \cup \mathcal{I}_{p}^{m}: x_{i, p} \notin \mathcal{C}_{p}$ move toward the containment area $\mathcal{C}_{p}$ defined by the leaders 
along the $p$-th component with a speed that is upper bounded by eqs. (70) and (78) and that they eventually reach $\mathcal{C}_{p}$ in finite-time $T$. Applying the same reasoning to all the $d$ components of the state, the result follows.

\section{APPENDIX B \\ PROOF OF THEOREM 5}

The result follows from the proof of Theorem 3 . In particular, since the edge set $\mathcal{E}(t)$ is assumed to vary only with respect to the time variable $t$ and not with respect to the state vector $\left[\mathbf{x}^{T} \mathbf{x}_{a}^{T}\right]^{T}$, and by assumption it changes only at instants of time which belong to a set of measure zero, it follows that such instants of time can be disregarded in the analysis and the structure of the set-valued Lie derivative in eq. (77) and the negative definiteness of the generalized time derivative in eq. (79) are not influenced as long as condition (20) is verified for all $t \notin \Omega$, i.e., (see eq. (79p)

$$
\frac{d}{d t}\left(V_{p}\left(\mathbf{x}_{p}\right)\right) \leq-\mu, \quad \forall t \notin \Omega,
$$

where

$$
\mu=-2 \alpha\left(\left(1-\frac{u_{\max }^{\ell}}{\alpha}\right)\left|\left(\mathcal{V}_{f}, \mathcal{V}_{\ell}\right)\right|-\left|\left(\mathcal{V}_{f}, \mathcal{V}_{a}\right)\right|\right)>2 \alpha
$$

Notice that this holds even if the parameters $r$ and $s$ change with time, as long as the condition 20 is satisfied.

A characterization of the convergence time can be obtained as done in Theorem 4 since the Lebesgue integral in eq. (33) over the time instants $t \in \Omega$ has no contribution because the set has zero measure and can hence be discarded. The result thus follows.

\section{REFERENCES}

[1] D. Panagou, D. M. Stipanovi, and P. G. Voulgaris, "Distributed Coordination Control for Multi-Robot Networks Using Lyapunov-Like Barrier Functions," IEEE Transactions on Automatic Control, vol. 61, no. 3, pp. 617-632, March 2016.

[2] A. Abdessameud, I. G. Polushin, and A. Tayebi, "Distributed Coordination of Dynamical Multi-Agent Systems Under Directed Graphs and Constrained Information Exchange," IEEE Transactions on Automatic Control, vol. 62, no. 4, pp. 1668-1683, April 2017.

[3] G. Li, D. St-Onge, C. Pinciroli, A. Gasparri, E. Garone, and G. Beltrame, "Decentralized progressive shape formation with robot swarms," Autonomous Robots, vol. 43, no. 6, pp. 1505-1521, 2019.

[4] J. Kallberg, "Designer Satellite Collisions from Covert Cyber War," Strategic Studies Quarterly, vol. 6, no. 1, pp. 124-136, 2012.

[5] D. Zhou, Z. Wang, and M. Schwager, "Agile Coordination and Assistive Collision Avoidance for Quadrotor Swarms Using Virtual Structures," IEEE Transactions on Robotics, vol. 34, no. 4, pp. 916-923, Aug 2018.

[6] A. Ajorlou, A. G. Aghdam, and A. Jadbabaie, "A connectivity preserving containment control strategy for unicycles with static leaders," in 2012 American Control Conference (ACC), June 2012, pp. 4186-4191.

[7] Y. Wang, Y. Song, D. J. Hill, and M. Krstic, "Prescribed-Time Consensus and Containment Control of Networked Multiagent Systems," IEEE Transactions on Cybernetics, vol. 49, no. 4, pp. 1138-1147, April 2019.

[8] M. Ji, G. Ferrari-Trecate, M. Egerstedt, and A. Buffa, "Containment Control in Mobile Networks," IEEE Transactions on Automatic Control, vol. 53, pp. 65-78, 2008.

[9] Y. Cao, W. Ren, and M. Egerstedt, "Distributed containment control with multiple stationary or dynamic leaders in fixed and switching directed networks," Automatica, vol. 48, no. 8, pp. 1586 - 1597, 2012.

[10] J. Fu, Y. Wan, G. Wen, and T. Huang, "Distributed Robust Global Containment Control of Second-Order Multiagent Systems With Input Saturation," IEEE Transactions on Control of Network Systems, vol. 6 , no. 4, pp. 1426-1437, Dec 2019.
[11] Y. Chen, S. Kar, and J. M. F. Moura, "Attack Resilient Distributed Estimation: A Consensus+Innovations Approach," in 2018 Annual American Control Conference (ACC), June 2018, pp. 1015-1020.

[12] H. Zhang and S. Sundaram, "Robustness of Information Diffusion Algorithms to Locally Bounded Adversaries," in 2012 American Control Conference (ACC), June 2012, pp. 5855-5861.

[13] H. J. LeBlanc, H. Zhang, X. Koutsoukos, and S. Sundaram, "Resilient Asymptotic Consensus in Robust Networks," IEEE Journal on Selected Areas in Communications, vol. 31, no. 4, pp. 766-781, April 2013.

[14] J. Usevitch and D. Panagou, "r-Robustness and (r, s)-robustness of circulant graphs," in 2017 IEEE 56th Annual Conference on Decision and Control (CDC), Dec 2017, pp. 4416-4421.

[15] Y. Wu and X. He, "Secure consensus control for multiagent systems with attacks and communication delays," IEEE/CAA Journal of Automatica Sinica, vol. 4, no. 1, pp. 136-142, Jan 2017.

[16] Y. Shang, "Consensus of Hybrid Multi-Agent Systems With Malicious Nodes," IEEE Transactions on Circuits and Systems II: Express Briefs, vol. 67, no. 4, pp. 685-689, April 2020.

[17] L. Guerrero-Bonilla, D. Saldaa, and V. Kumar, "Design Guarantees for Resilient Robot Formations on Lattices," IEEE Robotics and Automation Letters, vol. 4, no. 1, pp. 89-96, Jan 2019.

[18] S. M. Dibaji and H. Ishii, "Resilient consensus of second-order agent networks: Asynchronous update rules with delays," Automatica, vol. 81, pp. $123-132,2017$.

[19] H. J. LeBlanc and X. Koutsoukos, "Resilient First-Order Consensus and Weakly Stable, Higher Order Synchronization of Continuous-Time Networked Multiagent Systems," IEEE Transactions on Control of Network Systems, vol. 5, no. 3, pp. 1219-1231, Sep. 2018.

[20] K. Saulnier, D. Saldaa, A. Prorok, G. J. Pappas, and V. Kumar, "Resilient Flocking for Mobile Robot Teams," IEEE Robotics and Automation Letters, vol. 2, no. 2, pp. 1039-1046, April 2017.

[21] S. Zuo, F. L. Lewis, and A. Davoudi, "Resilient Output Containment of Heterogeneous Cooperative and Adversarial Multigroup Systems," IEEE Transactions on Automatic Control, vol. 65, no. 7, pp. 3104-3111, July 2020.

[22] Q. Li, L. Xia, and R. Song, "Output Resilient Containment Control of Heterogeneous Systems With Active Leaders Using Reinforcement Learning Under Attack Inputs," IEEE Access, vol. 7, pp. 162219$162228,2019$.

[23] C. Zhao, J. He, and J. Chen, "Resilient Consensus with Mobile Detectors Against Malicious Attacks," IEEE Transactions on Signal and Information Processing over Networks, vol. 4, no. 1, pp. 60-69, March 2018.

[24] S. M. Dibaji, M. Safi, and H. Ishii, "Resilient Distributed Averaging," in 2019 American Control Conference (ACC), 2019, pp. 96-101.

[25] Z. Li and H. Ji, "Finite-Time Consensus and Tracking Control of A Class of Nonlinear Multiagent Systems," IEEE Transactions on Automatic Control, vol. 63, no. 12, pp. 4413-4420, Dec 2018.

[26] P. Lin, W. Ren, and J. A. Farrell, "Distributed Continuous-Time Optimization: Nonuniform Gradient Gains, Finite-Time Convergence, and Convex Constraint Set," IEEE Transactions on Automatic Control, vol. 62 , no. 5, pp. 2239-2253, May 2017.

[27] L. Wang and F. Xiao, "Finite-Time Consensus Problems for Networks of Dynamic Agents," IEEE Transactions on Automatic Control, vol. 55, no. 4, pp. 950-955, 2010 .

[28] G. Chen, F. L. Lewis, and L. Xie, "Finite-time distributed consensus via binary control protocols," Automatica, vol. 47, no. 9, pp. 1962 - 1968, 2011.

[29] J. Cortés, "Finite-time convergent gradient flows with applications to network consensus," Automatica, vol. 42, no. 11, pp. 1993-2000, 2006.

[30] Q. Hui, W. M. Haddad, and S. P. Bhat, "Finite-time semistability, Filippov systems, and consensus protocols for nonlinear dynamical networks with switching topologies," Nonlinear Analysis: Hybrid Systems, vol. 4, no. 3, pp. $557-573,2010$.

[31] Y. Wang, Y. Song, D. J. Hill, and M. Krstic, "Prescribed finite time consensus of networked multi-agent systems," in 2017 IEEE 56th Annual Conference on Decision and Control (CDC), Dec 2017, pp. 4088-4093.

[32] S. Sundaram and B. Gharesifard, "Distributed Optimization Under Adversarial Nodes," IEEE Transactions on Automatic Control, vol. 64, no. 3, pp. 1063-1076, March 2019.

[33] H. Hong, W. Yu, J. Fu, and X. Yu, "Finite-Time Connectivity-Preserving Consensus for Second-Order Nonlinear Multiagent Systems," IEEE Transactions on Control of Network Systems, vol. 6, no. 1, pp. 236248, March 2019

[34] X. Chu, Z. Peng, G. Wen, and A. Rahmani, "Robust fixed-time consensus tracking with application to formation control of unicycles," IET Control Theory Applications, vol. 12, no. 1, pp. 53-59, 2018. 
[35] C. Sun, G. Hu, L. Xie, and M. Egerstedt, "Robust finite-time connectivity preserving consensus tracking and formation control for multi-agent systems," in 2017 American Control Conference (ACC), May 2017, pp. 1990-1995.

[36] M. Franceschelli, A. Giua, and A. Pisano, "Finite-Time Consensus on the Median Value With Robustness Properties," IEEE Transactions on Automatic Control, vol. 62, no. 4, pp. 1652-1667, 2017.

[37] F. Pasqualetti, A. Bicchi, and F. Bullo, "Consensus Computation in Unreliable Networks: A System Theoretic Approach," IEEE Transactions on Automatic Control, vol. 57, no. 1, pp. 90-104, 2012.

[38] J. Li, E. Elhamifar, I.-J. Wang, and R. Vidal, "Consensus with robustness to outliers via distributed optimization," in 49th IEEE Conference on Decision and Control (CDC), 2010, pp. 2111-2117.

[39] M. Franceschelli, A. Giua, and A. Pisano, "Finite-time consensus on the median value by discontinuous control," in 2014 American Control Conference (ACC), 2014, June 2014, pp. 946-951.

[40] A. Bacciotti and F. Ceragioli, "Stability and Stabilization of Discontinuous Systems and Nonsmooth Lyapunov Functions," ESAIM Control, Optimisation and Calculus of Variations, no. 4, pp. 361-376, 1999.

[41] B. Paden and S. Sastry, "A calculus for computing Filippov's differential inclusion with application to the variable structure control of robot manipulators," IEEE Transactions on Circuits and Systems, vol. 34, no. 1, pp. 73-82, 1987.

[42] D. Shevitz and B. Paden, "Lyapunov stability theory of nonsmooth systems," IEEE Transactions on Automatic Control, vol. 39, no. 9, pp. 1910-1914, 1994.

[43] J. Cortes, "Discontinuous dynamical systems," IEEE Control Systems Magazine, vol. 28, no. 3, pp. 36-73, 2008

[44] F. Clarke, Optimization and Nonsmooth Analysis. Wiley \& Sons, New York, 1983.

[45] A. Filippov, Differential Equations with Discontinuous Righthand Sides, Kluwer Academic Publishers, Dordrecht, The Netherlands, 1988.

[46] M. Franceschelli and A. Gasparri, "Gossip-Based Centroid and Common Reference Frame Estimation in Multiagent Systems," IEEE Transactions on Robotics, vol. 30, no. 2, pp. 524-531, 2014.

[47] M. Santilli, M. Franceschelli, and A. Gasparri, "Robust Containment Control in Multi-Agent Systems with Common Coordinate Frames and Bearing Angle Measurements," in 2019 IEEE 58th Conference on Decision and Control (CDC), 2019, pp. 3710-3717.

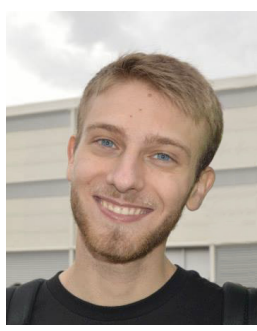

Matteo Santilli received the bachelor and master's degrees (cum laude) in computer science engineering and in computer science and automation engineering from Roma Tre University, in 2015 and 2017, respectively. In 2021 he received the Ph.D. degree in automation and computer science with the Department of Engineering at Roma Tre University, where he currently serves as postdoctoral research fellow. He spent a visiting period at Virginia Polytechnic Institute and State University, Blacksburg, VA, USA. His research interests include nonlinear multi-agent systems, resilient control problems, and distributed optimization.

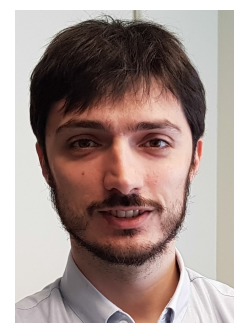

Mauro Franceschelli (M'11) is Senior Assistant Professor (RTD-B) at the Department of Electrical and Electronic Engineering, University of Cagliari, Italy, since 2019. He received the Laurea degree in Electronic Engineering with honors "cum laude" in 2007 and the $\mathrm{PhD}$ degree in 2011 from the University of Cagliari. He spent visiting periods at the Georgia Institute of Technology (GaTech), and the University of California at Santa Barbara (UCSB), USA.

In 2013 he received a fellowship from the $\mathrm{Na}$ tional Natural Science Foundation of China (NSFC), at Xidian University, Xi' an, China. In 2015 he was awarded a position of Assistant Professor funded by the Italian Ministry of Education, University and Research (MIUR) under the 2014 call "Scientific Independence of Young Researchers" (SIR) with project "CoNetDomeSys". He is Associate Editor for the IEEE Transactions on Automation Science and Engineering (T-ASE) since 2021. He is member of the Conference Editorial Board (CEB) of the IEEE Control Systems Society (CSS) since 2019. He serves as Associate Editor for the IEEE Conference on Automation Science and Engineering (CASE) since 2015, the IEEE American Control Conference (ACC) since 2019 and IEEE Conference on Decision and Control since 2020. He is member of the IEEE RAS Technical Committee on Multi-robot Systems and IEEE CSS Technical Committee on Networks and Communications. His research interests include consensus problems, gossip algorithms, multi-agent systems, multi-robot systems, distributed optimization and electric demand side management.

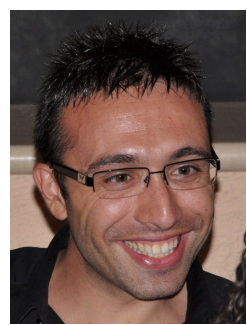

Andrea Gasparri (M'09, SM'19) received the cum laude Laurea degree in computer science in 2004 and the Ph.D. degree in computer science and automation in 2008 from Roma Tre University, where he currently serves as Professor in the Department of Engineering. His research interests are in the areas of robotics, sensor networks, networked multi-agent systems and precision agriculture. $\mathrm{He}$ is a member of the Steering Committee for the IEEE RAS Technical Committee on Multirobot Systems since 2014 and of the IEEE CSS Technical Committee on Networks and Communications since 2015. He is Associate Editor of the IEEE Transactions on Cybernetics since 2017. He is Associate Editor of the IEEE Transactions on Control of Network Systems since 2021. Prof Gasparri received the Italian Grant FIRB Futuro in Ricerca 2008 for the project Networked Collaborative Team of Autonomous Robots funded by the Italian Ministry of Research and Education. Prof. Gasparri is currently the coordinator of the project "PANTHEON" focused on robotics for precision farming supported by the European Community within the H2020 framework (under grant agreement number 774571) and of the project "CANOPIES" focused on on the development of a collaborative paradigm for human workers and multi-robot teams in precision agriculture systems within the H2020 framework (under grant agreement number 101016906). 